\title{
A New Asymptotic Perturbation Theory with Applications to Models of Massless Quantum Fields
}

\author{
Asao Arai \\ Department of Mathematics, Hokkaido University \\ Sapporo 060-0810 \\ Japan \\ E-mail: arai@math.sci.hokudai.ac.jp
}

December 7, 2012

\begin{abstract}
Let $H_{0}$ and $H_{\mathrm{I}}$ be a self-adjoint and a symmetric operator on a complex Hilbert space, respectively, and suppose that $H_{0}$ is bounded below and the infimum $E_{0}$ of the spectrum of $H_{0}$ is a simple eigenvalue of $H_{0}$ which is not necessarily isolated. In this paper, we present a new asymptotic perturbation theory for an eigenvalue $E(\lambda)$ of the operator $H(\lambda):=H_{0}+\lambda H_{\mathrm{I}}(\lambda \in \mathbb{R} \backslash\{0\})$ satisfying $\lim _{\lambda \rightarrow 0} E(\lambda)=E_{0}$. The point of the theory is in that it covers also the case where $E_{0}$ is a non-isolated eigenvalue of $H_{0}$. Under a suitable set of assumptions, we derive an asymptotic expansion of $E(\lambda)$ up to an arbitrary finite order of $\lambda$ as $\lambda \rightarrow 0$. We apply the abstract results to a model of massless quantum fields, called the generalized spinboson model (A. Arai and M. Hirokawa, J. Funct. Anal. 151 (1997), 455-503) and show that the ground state energy of the model has asymptotic expansions in the coupling constant $\lambda$ as $\lambda \rightarrow 0$.
\end{abstract}

Keywords: asymptotic perturbation theory, embedded eigenvalue, generalized spin-boson model, ground state energy, non-isolated eigenvalue, massless quantum field

Mathematics Subject Classification 2010: 47A55, 81Q10, 81Q15

\section{Introduction}

As is well known, in quantum mechanics, a perturbation theory is useful to calculate approximately various quantities (e.g., energy levels of an atom) under the condition that the relevant perturbation is "small". In the standard analytic perturbation theory ([15, 
Chapter 7], [18, §XII.2]) and the standard asymptotic perturbation theory ([15, Chapter 8], [18, §XII.3]), however, only perturbations of an isolated eigenvalue are considered. But the perturbation problem of a non-isolated eigenvalue (typically an embedded eigenvalue) naturally appears in many-body quantum systems and models of massless quantum fields. For the former, dilation analytic methods (e.g., [18, §XII.6]) have been shown to be useful. For the latter, Bach, Fröhlich and Sigal $[8,9])$ have developed a method exploring a renormalization group idea combined with the Feshbach map. Recently analyticity in the coupling constant for the ground state energy ${ }^{1}$ for some concrete models of massless quantum fields (in which the ground state energy of the unperturbed system under consideration is an embedded eigenvalue) has been proved $[1,2,12,13]$. These results are very nice, but, they may be model-dependent. From a general mathematical point of view, it would be valuable to investigate to what extent it is possible to develop a modelindependent general perturbation theory for non-isolated eigenvalues in such a way that, at least, it can be applied to the perturbation of a non-isolated ground state energy in models of massless quantum fields. To our best knowledge, such a general perturbation theory is still missing. With this motivation, we present, in this paper, as a first step, a new asymptotic perturbation theory which may have applications to models of a quantum system interacting with a massless quantum field. In the present paper, we do not discuss possible analytic extensions of our asymptotic perturbation theory. This aspect is left for future studies.

The outline of the present paper is as follows. In Section 2, we define an operator for which we give a perturbation theoretical consideration and derive some facts which are bases for the asymptotic perturbation theory developed in this paper. The operator is of the following form:

$$
H(\lambda):=H_{0}+\lambda H_{\mathrm{I}}
$$

where $H_{0}$ (resp. $H_{\mathrm{I}}$ ) is a symmetric (resp. linear) operator acting on a complex Hilbert space $\mathcal{H}$ such that $D\left(H_{0}\right) \cap D\left(H_{\mathrm{I}}\right) \neq\{0\}$ and $\lambda \in \mathbb{R} \backslash\{0\}$ is the coupling constant (perturbation parameter). We are mainly interested in the case where $H_{0}$ has a simple eigenvalue $E_{0}$ which is not isolated. A basic new idea which allows one to treat such a case comes from the so-called Brillouin-Wigner perturbation theory [10, 19, 20], which, on an informal (heuristic) level, may be more elaborate than the Rayleigh-Schrödinger perturbation theory. We use the Brillouin-Wigner perturbation theory in a "non-perturbative" way: We derive a simultaneous (non-perturbative, closed) equation for an eigenvalue $E$ and an eigenvector $\Psi$ of $H(\lambda)(H(\lambda) \Psi=E \Psi)$ under a condition for $E$ (Proposition 2.1). This equation is one of the basic starting points in our analysis. Another basic fact we derive in Section 2 is an upper bound for the infimum $\mathcal{E}_{0}(\lambda)$ of the numerical range of $H(\lambda)$

\footnotetext{
${ }^{1}$ For a mathematical definition, see Remark 2.4.
} 
in the case where $H_{0}$ is self-adjoint, bounded below and $H_{\mathrm{I}}$ is symmetric (Theorem 2.7). This aspect may have an independent interest. As a corollary to this fact, a sufficient condition for $\mathcal{E}_{0}(\lambda)$ to be in the resolvent set of $H_{0}$ is given (Corollary 2.10).

In Section 3, under additional assumptions, we derive an asymptotic expansion for the eigenvalue $E=E(\lambda)$ of $H(\lambda)$ up to the second order of $\lambda$ as $\lambda \rightarrow 0$ (Theorem 3.5).

In Section 4, for each natural number $N \geq 2$, under stronger assumptions, we derive an asymptotic expansion for $E(\lambda)$ up to the $N$-th order of $\lambda$ as $\lambda \rightarrow 0$ (Theorem 4.1).

The last section is devoted to an application of Theorem 4.1 to the generalized spinboson (GSB) model [5], which describes an abstract quantum system interacting with a Bose field. We first show that the infimum of the spectrum of the Hamiltonian of the GSB model is an even function of the coupling constant $\lambda$ (Theorem 5.1). Then we prove that, under a set of suitable assumptions which, in the case where the Bose field is massless, requires a stronger infrared regular condition for the momentum cutoff functions of the Bose field in the interaction part, the ground state energy of the Hamiltonian of the GSB model has an asymptotic expansion in the coupling constant $\lambda$ up to an arbitrary finite order of $\lambda$ with all the coefficients of odd powers of $\lambda$ vanishing (Theorem 5.17).

\section{Baic Facts}

We denote the inner product and the norm of the Hilbert space $\mathcal{H}$ by $\langle\cdot, \cdot\rangle$ (complex linear in the second variable) and $\|\cdot\|$ respectively. For a linear operator $A$ on a Hilbert space, we use the following notation:

(i) $D(A)$ : The domain of $A$.

(ii) ker $A$ : The kernel of $A$.

(iii) $\rho(A)$ : The resolvent set of $A$.

(iv) $\sigma(A):=\mathbb{C} \backslash \rho(A)$ : The spectrum of $A$.

(v) $\sigma_{\mathrm{p}}(A)$ : The point spectrum of $A$ (the set of eigenvalues of $A$ ).

In this section, we derive some fundamental facts on the operator $H(\lambda)$ defined by (1.1). As already mentioned in Introduction, one of the basic hypotheses in our theory is the following:

(H.1) The operator $H_{0}$ has a simple eigenvalue $E_{0}$.

By the symmetry of $H_{0}, E_{0}$ is a real number. We fix a normalized eigenvector $\Psi_{0}$ of $H_{0}$ with eigenvalue $E_{0}$ :

$$
H_{0} \Psi_{0}=E_{0} \Psi_{0}, \quad\left\|\Psi_{0}\right\|=1 .
$$


Let $P_{0}$ be the orthogonal projection onto the eigenspace

$$
\mathcal{H}_{0}:=\left\{\alpha \Psi_{0} \mid \alpha \in \mathbb{C}\right\}
$$

of $H_{0}$ with eigenvalue $E_{0}$. Then

$$
Q_{0}:=I-P_{0}
$$

is the orthogonal projection onto the orthogonal complement $\mathcal{H}_{0}^{\perp}$ of $\mathcal{H}_{0}$. Since $H_{0}$ is symmetric, $H_{0}$ is reduced by both $\mathcal{H}_{0}$ and $\mathcal{H}_{0}^{\perp}$, i.e., $P_{0} H_{0} \subset H_{0} P_{0}$. We denote by $H_{0}^{\prime}$ the reduced part of $H_{0}$ to $\mathcal{H}_{0}^{\perp}$.

\subsection{Simultaneous equation for an eigenvalue and an eigenvector of $H(\lambda)$}

We say that two vectors $\Psi$ and $\Phi$ in $\mathcal{H}$ overlap (or $\Psi$ overlaps with $\Phi$ ) if $\langle\Psi, \Phi\rangle \neq 0$. Also we say that a vector $\Psi \in \mathcal{H}$ overlaps with a non-empty subset $\mathcal{D} \subset \mathcal{H}$ if there exists a vector $\Phi \in \mathcal{D}$ which overlaps with $\Psi$.

The following proposition is a rigorous non-perturbative formulation of ideas behind the Brillouin-Wigner perturbation method [10, 19] (cf. [20, §3.1]):

Proposition 2.1 Let $E_{0}, \Psi_{0}$ and $Q_{0}$ be as above, and $\lambda \in \mathbb{R} \backslash\{0\}$ be fixed. Let $E$ be a complex number with $E \notin \sigma_{\mathrm{p}}\left(H_{0}^{\prime}\right)$ (hence the inverse $\left(E-H_{0}^{\prime}\right)^{-1}$ of $E-H_{0}^{\prime}$ exists on $\mathcal{H}_{0}^{\perp}$, being not necessarily bounded) and $\Psi \in D(H(\lambda))$.

(i) If $E$ is an eigenvalue of $H(\lambda)$ (i.e., $E \in \sigma_{\mathrm{p}}(H(\lambda))$ ) and $\Psi_{0}$ overlaps with the eigenspace $\operatorname{ker}(H(\lambda)-E)$ of $H(\lambda)$ with eigenvalue $E$, then there exists a non-zero vector $\Psi \in \operatorname{ker}(H(\lambda)-E)$ such that $Q_{0} H_{\mathrm{I}} \Psi \in D\left(\left(E-H_{0}^{\prime}\right)^{-1}\right)$ and

$$
\begin{aligned}
& E=E_{0}+\lambda\left\langle\Psi_{0}, H_{\mathrm{I}} \Psi\right\rangle, \\
& \Psi=\Psi_{0}+\lambda\left(E-H_{0}^{\prime}\right)^{-1} Q_{0} H_{\mathrm{I}} \Psi .
\end{aligned}
$$

(ii) Conversely, if $E$ and $\Psi$ satisfy (2.4) and (2.5), then $E$ is an eigenvalue of $H(\lambda)$ and $\Psi$ is an eigenvector of $H(\lambda)$ with eigenvalue $E$ (i.e., $\Psi \in \operatorname{ker}(H(\lambda)-E) \backslash\{0\}$ ), overlapping with $\Psi_{0}$.

Proof. (i) By the present assumption, there exists a vector $\Xi$ in $\operatorname{ker}(H(\lambda)-E)$ such that $\left\langle\Psi_{0}, \Xi\right\rangle \neq 0$. We have $\left\langle\Psi_{0}, H(\lambda) \Xi\right\rangle=E\left\langle\Psi_{0}, \Xi\right\rangle$. The left hand side is equal to $E_{0}\left\langle\Psi_{0}, \Xi\right\rangle+\lambda\left\langle\Psi_{0}, H_{\mathrm{I}} \Xi\right\rangle$. Hence $E\left\langle\Psi_{0}, \Xi\right\rangle=E_{0}\left\langle\Psi_{0}, \Xi\right\rangle+\lambda\left\langle\Psi_{0}, H_{\mathrm{I}} \Xi\right\rangle$. Since $\left\langle\Psi_{0}, \Xi\right\rangle \neq 0$, we obtain (2.4) with $\Psi:=\Xi /\left\langle\Psi_{0}, \Xi\right\rangle$. 
To prove (2.5), we note that $\left\langle\Psi_{0}, \Psi\right\rangle=1$. Hence, by the projection theorem, there exists a unique vector $\Phi \in \mathcal{H}_{0}^{\perp}$ such that

$$
\Psi=\Psi_{0}+\Phi
$$

Since both $\Psi$ and $\Psi_{0}$ are in $D\left(H_{0}\right), \Phi$ is in $D\left(H_{0}\right)$. Hence

$$
\lambda H_{\mathrm{I}} \Psi=\left(E-H_{0}\right) \Psi=\left(E-E_{0}\right) \Psi_{0}+\left(E-H_{0}\right) \Phi .
$$

By the fact that $Q_{0} \Psi_{0}=0$ and $Q_{0}\left(E-H_{0}\right) \Phi=\left(E-H_{0}^{\prime}\right) \Phi$, we have

$$
\lambda Q_{0} H_{\mathrm{I}} \Psi=\left(E-H_{0}^{\prime}\right) \Phi
$$

This means that $Q_{0} H_{\mathrm{I}} \Psi \in D\left(\left(E-H_{0}^{\prime}\right)^{-1}\right)$ and $\Phi=\lambda\left(E-H_{0}^{\prime}\right)^{-1} Q_{0} H_{\mathrm{I}} \Psi$. Thus (2.5) holds.

(ii) Eq.(2.5) implies that $\Psi \neq 0$ and

$$
\left(E-H_{0}\right) \Psi=\left(E-E_{0}\right) \Psi_{0}+\lambda Q_{0} H_{\mathrm{I}} \Psi
$$

Hence

$$
\begin{aligned}
(E-H(\lambda)) \Psi & =\left(E-E_{0}\right) \Psi_{0}+\lambda Q_{0} H_{\mathrm{I}} \Psi-\lambda H_{\mathrm{I}} \Psi=\left(E-E_{0}\right) \Psi_{0}-\lambda P_{0} H_{\mathrm{I}} \Psi \\
& =\left(E-E_{0}\right) \Psi_{0}-\lambda\left\langle\Psi_{0}, H_{\mathrm{I}} \Psi\right\rangle \Psi_{0} .
\end{aligned}
$$

But, by (2.4), the last vector is zero. Hence $\Psi \in \operatorname{ker}(H(\lambda)-E) \backslash\{0\}$. Taking the inner product of $\Psi_{0}$ with $(2.5)$, we have $\left\langle\Psi_{0}, \Psi\right\rangle=1$. Hence $\Psi$ overlaps with $\Psi_{0}$.

Remark 2.2 Proposition 2.1-(ii) may be used to establish an existence theorem of an eigenvalue of $H(\lambda)$. But, in the present paper, we do not go into considerations of this aspect.

Remark 2.3 Instead of $H(\lambda)$ given by (1.1), one can also consider a more general operator of the following form:

$$
H(\lambda)=H_{0}+\sum_{i=1}^{n} \lambda^{i} H_{i}
$$

with $n \in \mathbb{N}$ arbitrary and $H_{i}(i=1, \ldots, n)$ being a linear operator on $\mathcal{H}$. It is obvious that, in this case, Proposition 2.1 holds with $\lambda H_{\mathrm{I}}$ replaced by $\sum_{i=1}^{n} \lambda^{i} H_{i}$. Hence, for a theory which is developed based on Proposition 2.1, as is done below, it may be enough to consider the case $n=1$. Results in the case $n=1$ would easily be translated into the case $n \geq 2$ by the replacement of $\lambda H_{\mathrm{I}}$ with $\sum_{i=1}^{n} \lambda^{i} H_{i}$. 


\subsection{Upper bound for the infimum of the numerical range of $H(\lambda)$}

In this subsection, in addition to (H.1), we assume the following:

(H.2) The operator $H_{0}$ is self-adjoint and $E_{0}=\inf \sigma\left(H_{0}\right)$.

Remark 2.4 In general, if a self-adjoint operator $H$ on a Hilbert space is bounded below, then

$$
E_{\min }(H):=\inf \sigma(H)
$$

is called the lowest or minimal energy of $H$. If $E_{\min }(H)$ is an eigenvalue of $H$, then $H$ is said to have a ground state and $E_{\min }(H)$ is called the ground state energy of $H$. In this case, a non-zero vector of $\operatorname{ker}\left(H-E_{\min }(H)\right)$ is called a ground state of $H$. Assumptions (H.1) and (H.2) mean that $H_{0}$ has a unique ground state $\Psi_{0}$ (up to constant multiples) with $E_{0}$ being the ground state energy.

Under assumptions (H.1) and (H.2), $H_{0}^{\prime}-E_{0}$ is an injective and non-negative selfadjoint operator on $\mathcal{H}_{0}^{\perp}$. Hence the inverse operator $\left(H_{0}^{\prime}-E_{0}\right)^{-1}$ exists on $\mathcal{H}_{0}^{\perp}$ and is a non-negative self-adjoint operator.

Remark 2.5 If $E_{0}$ is an isolated eigenvalue of $H_{0}$, then $\left(H_{0}^{\prime}-E_{0}\right)^{-1}$ is bounded. This case is a situation where the standard analytic perturbation theory and the standard asymptotic perturbation theory are formulated. But the case where $E_{0}$ is not an isolated eigenvalue of $H_{0}$ is out of those perturbation theories. In addition, in this case, $\left(H_{0}^{\prime}-E_{0}\right)^{-1}$ is unbounded and hence one has to be careful about domains of operators.

If $H_{\mathrm{I}}$ is symmetric, then, for all $\Psi \in D(H(\lambda)),\langle\Psi, H(\lambda) \Psi\rangle$ is a real number. Hence one can define

$$
\left.\mathcal{E}_{0}(\lambda):=\inf _{\Psi \in D(H(\lambda)),\|\Psi\|=1}\langle\Psi, H(\lambda)) \Psi\right\rangle \quad(\lambda \in \mathbb{R}),
$$

the infimum of the numerical range of $H(\lambda)$, which is a finite real number or $-\infty$, where, for $\lambda=0$, we set $H(0):=H_{0}$ so that, by the variational principle

$$
\varepsilon_{0}(0)=E_{0}
$$

Remark 2.6 If $H(\lambda)$ is self-adjoint and bounded below, then $\mathcal{E}_{0}(\lambda)=E_{\min }(H(\lambda))$ by the variational principle. But, in general, $\mathcal{E}_{0}(\lambda)$ is not necessarily an eigenvalue of $H(\lambda)$.

Theorem 2.7 Assume (H.1) and (H.2). Suppose that $H_{\mathrm{I}}$ is symmetric and

$$
\left.\Psi_{0} \in D\left(H_{0}\left(H_{0}^{\prime}-E_{0}\right)^{-1} Q_{0} H_{\mathrm{I}}\right) \cap D\left(H_{\mathrm{I}}\left(H_{0}^{\prime}-E_{0}\right)^{-1} Q_{0} H_{\mathrm{I}}\right)\right) .
$$


Let

$$
\begin{aligned}
& N_{0}:=\left\|\left(H_{0}^{\prime}-E_{0}\right)^{-1} Q_{0} H_{\mathrm{I}} \Psi_{0}\right\|^{2}, \\
& a:=\left\langle Q_{0} H_{\mathrm{I}} \Psi_{0},\left(H_{0}^{\prime}-E_{0}\right)^{-1} Q_{0} H_{\mathrm{I}} \Psi_{0}\right\rangle \\
& b:=\left\langle\left(H_{0}^{\prime}-E_{0}\right)^{-1} Q_{0} H_{\mathrm{I}} \Psi_{0}, H_{\mathrm{I}}\left(H_{0}^{\prime}-E_{0}\right)^{-1} Q_{0} H_{\mathrm{I}} \Psi_{0}\right\rangle .
\end{aligned}
$$

Then, for all $\lambda \in \mathbb{R}$,

$$
\mathcal{E}_{0}(\lambda) \leq E_{0}+\frac{1}{1+N_{0} \lambda^{2}}\left(\left\langle\Psi_{0}, H_{\mathrm{I}} \Psi_{0}\right\rangle \lambda-a \lambda^{2}+b \lambda^{3}\right) .
$$

Proof. By (2.7), we have for all $\Psi \in D(H(\lambda)) \backslash\{0\}$

$$
\varepsilon_{0}(\lambda) \leq \frac{\langle\Psi, H(\lambda) \Psi\rangle}{\|\Psi\|^{2}}
$$

As the vector $\Psi$, we take

$$
\Psi_{1}:=\Psi_{0}-\lambda\left(H_{0}^{\prime}-E_{0}\right)^{-1} Q_{0} H_{\mathrm{I}} \Psi_{0}
$$

Then, by (2.9), $\Psi_{1} \in D(H(\lambda))$ and we have

$$
\left\|\Psi_{1}\right\|^{2}=1+N_{0} \lambda^{2}
$$

where $N_{0}$ is defined by (2.10). Since $\Psi_{0}$ is orthogonal to $\operatorname{Ran}\left(Q_{0}\right)$ (the range of $\left.Q_{0}\right)$ and $H_{0}$ maps $D\left(H_{0}\right) \cap \operatorname{Ran}\left(Q_{0}\right)$ to $\operatorname{Ran}\left(Q_{0}\right)$, it follows that

$$
\left\langle\Psi_{1}, H_{0} \Psi_{1}\right\rangle=E_{0}+\lambda^{2}\left\langle\left(H_{0}^{\prime}-E_{0}\right)^{-1} Q_{0} H_{\mathrm{I}} \Psi_{0}, H_{0}\left(H_{0}^{\prime}-E_{0}\right)^{-1} Q_{0} H_{\mathrm{I}} \Psi_{0}\right\rangle .
$$

Using the identity

$$
H_{0}\left(H_{0}^{\prime}-E_{0}\right)^{-1} Q_{0}=Q_{0}+E_{0}\left(H_{0}^{\prime}-E_{0}\right)^{-1} Q_{0},
$$

we obtain

$$
\left\langle\Psi_{1}, H_{0} \Psi_{1}\right\rangle=E_{0}\left\|\Psi_{1}\right\|^{2}+a \lambda^{2}
$$

with $a$ defined by (2.11). Similarly we have

$$
\left\langle\Psi_{1}, H_{\mathrm{I}} \Psi_{1}\right\rangle=\left\langle\Psi_{0}, H_{\mathrm{I}} \Psi_{0}\right\rangle-2 a \lambda+b \lambda^{2}
$$

with $b$ given by (2.12). Hence we obtain

$$
\left\langle\Psi_{1}, H(\lambda) \Psi_{1}\right\rangle=E_{0}\left\|\Psi_{1}\right\|^{2}+\left\langle\Psi_{0}, H_{\mathrm{I}} \Psi_{0}\right\rangle \lambda-a \lambda^{2}+b \lambda^{3} .
$$

Hence, by (2.14) and (2.15), we obtain (2.13). 
Remark 2.8 Since $\left(H_{0}^{\prime}-E_{0}\right)^{-1}$ is non-negative as mentioned above, it follows that $a \geq 0$. It is easy to see that $a>0$ if and only if $Q_{0} H_{\mathrm{I}} \Psi_{0} \neq 0$.

Remark 2.9 The choice of the trial vector $\Psi_{1}$ in the proof just made is motivated by (2.4) and (2.5). Heuristically $\Psi_{1}$ may be an "approximate" solution of (2.5) in the first order of $\lambda$. Indeed, by iterating (2.5), we obtain

$$
\Psi=\Psi_{0}+\sum_{n=1}^{N} \lambda^{n}\left(\left(E-H_{0}^{\prime}\right)^{-1} Q_{0} H_{\mathrm{I}}\right)^{n} \Psi_{0}+\lambda^{N+1}\left(\left(E-H_{0}^{\prime}\right)^{-1} Q_{0} H_{\mathrm{I}}\right)^{N+1} \Psi,
$$

provided that $\Psi_{0} \in \cap_{n=1}^{N} D\left(\left(\left(E_{0}-H_{0}^{\prime}\right)^{-1} Q_{0} H_{\mathrm{I}}\right)^{n}\right)(N \in \mathbb{N})$ (then, by induction in $N$, one can show that $\left.\Psi \in D\left(\left(\left(E-H_{0}^{\prime}\right)^{-1} Q_{0} H_{\mathrm{I}}\right)^{N+1}\right)\right)$. Hence

$$
\Psi_{N}:=\Psi_{0}+\sum_{n=1}^{N} \lambda^{n}\left(\left(E_{0}-H_{0}^{\prime}\right)^{-1} Q_{0} H_{\mathrm{I}}\right)^{n} \Psi_{0}
$$

may be an "approximate" solution of (2.5) up to the $N$-th order of $\lambda$. Using the vector $\Psi_{N}(N \geq 2)$ as a trial vector, one may derive an inequality better than (2.13). But, in this paper, we do not go into the details.

Corollary 2.10 Under the same assumption as in Theorem 2.7, consider the case where

$$
\left|\left\langle\Psi_{0}, H_{\mathrm{I}} \Psi_{0}\right\rangle\right|<|\lambda|(a-b \lambda) .
$$

Then

$$
\varepsilon_{0}(\lambda)<E_{0}
$$

In particular, $\mathcal{E}_{0}(\lambda) \in \rho\left(H_{0}\right)$.

Proof. By condition (2.17), we have

$$
\left\langle\Psi_{0}, H_{\mathrm{I}} \Psi_{0}\right\rangle \lambda-a \lambda^{2}+b \lambda^{3}=\lambda\left\{\left\langle\Psi_{0}, H_{\mathrm{I}} \Psi_{0}\right\rangle-\lambda(a-b \lambda)\right\}<0
$$

Hence (2.13) implies (2.18). By (H.2), $\left(-\infty, E_{0}\right) \subset \rho\left(H_{0}\right)$. Hence $\mathcal{E}_{0}(\lambda) \in \rho\left(H_{0}\right)$ for all $\lambda$ obeying (2.17).

Remark 2.11 If

$$
\left\langle\Psi_{0}, H_{\mathrm{I}} \Psi_{0}\right\rangle=0
$$

and

$$
\lambda \neq 0, \quad b \lambda<a,
$$

then (2.17) holds and hence (2.18) too. 


\section{Asymptotic Expansion up to the Second Order in $\lambda$}

Let $E_{0}, \Psi_{0}$ and $Q_{0}$ be as in Section 2. In this section, in addition to (H.1), we assume the following:

(H.3) (i) $\Psi_{0} \in D\left(H_{0}\right) \cap D\left(H_{\mathrm{I}}\right)$.

(ii) There exists a constant $r>0$ such that, for all $\lambda$ in the set

$$
\mathbb{I}_{r}^{\times}:=(-r, 0) \cup(0, r),
$$

$H(\lambda)$ has an eigenvalue $E(\lambda)$ such that $E(\lambda) \notin \sigma_{\mathrm{p}}\left(H_{0}^{\prime}\right)$ and $\Psi_{0}$ overlaps with $\operatorname{ker}(H(\lambda)-E(\lambda))$.

Under assumptions (H.1) and (H.3), it follows from Proposition 2.1 that, for each $\lambda \in \mathbb{I}_{r}^{\times}$, there exists a non-zero vector $\Psi(\lambda) \in \operatorname{ker}(H(\lambda)-E(\lambda))$ satisfying

$$
\begin{aligned}
& E(\lambda)=E_{0}+\lambda\left\langle\Psi_{0}, H_{\mathrm{I}} \Psi(\lambda)\right\rangle, \\
& \Psi(\lambda)=\Psi_{0}+\Phi(\lambda),
\end{aligned}
$$

where

$$
\Phi(\lambda):=\lambda\left(E(\lambda)-H_{0}^{\prime}\right)^{-1} Q_{0} H_{\mathrm{I}} \Psi(\lambda) \in \mathcal{H}_{0}^{\perp} .
$$

Also we assume the following:

(H.4) $\lim _{\lambda \rightarrow 0}\|\Psi(\lambda)\|=1$.

By (3.2), we have

$$
\|\Psi(\lambda)\|^{2}=1+\|\Phi(\lambda)\|^{2}
$$

Hence (H.4) implies that

$$
\lim _{\lambda \rightarrow 0} \Phi(\lambda)=0
$$

Therefore

$$
\lim _{\lambda \rightarrow 0} \Psi(\lambda)=\Psi_{0}
$$

Under the framework described above, an elementary fact on the asymptotics for $E(\lambda)$ in $\lambda$ is:

Theorem 3.1 Assume (H.1), (H.3) and (H.4). Then,

$$
E(\lambda)=E_{0}+\lambda\left\langle\Psi_{0}, H_{\mathrm{I}} \Psi_{0}\right\rangle+o(\lambda) \quad(\lambda \rightarrow 0) .
$$

In particular,

$$
\lim _{\lambda \rightarrow 0} E(\lambda)=E_{0}
$$


Proof. We have by (3.1) and (3.2)

$$
E(\lambda)=E_{0}+\lambda\left\langle\Psi_{0}, H_{\mathrm{I}} \Psi_{0}\right\rangle+\lambda\left\langle H_{\mathrm{I}} \Psi_{0}, \Phi(\lambda)\right\rangle
$$

Hence, by (3.5), we have

$$
\frac{E(\lambda)-E_{0}-\lambda\left\langle\Psi_{0}, H_{\mathrm{I}} \Psi_{0}\right\rangle}{\lambda}=\left\langle H_{\mathrm{I}} \Psi_{0}, \Phi(\lambda)\right\rangle \rightarrow 0 \quad(\lambda \rightarrow 0) .
$$

Thus (3.7) holds.

Remark 3.2 Eq.(3.8) shows the continuity of $E(\lambda)$ at $\lambda=0$. In the case where (H.1) and (H.2) hold and $H(\lambda)$ is a bounded below self-adjoint operator for all $\lambda \in \mathbb{I}_{r}^{\times}$, one can prove the continuity of $\mathcal{E}_{0}(\lambda)$ in $\lambda \in \mathbb{I}_{r}^{\times}$under a condition which does not assume that $\mathcal{E}_{0}(\lambda)$ is an eigenvalue of $H(\lambda)[6$, Lemma 2.1].

To develop an asymptotic perturbation theory for $E(\lambda)$ further, we still need an additional assumption:

(H.5) For all $\lambda \in \mathbb{I}_{r}^{\times}, E(\lambda)$ is real, satisfying $E(\lambda)<E_{0}$.

Remark 3.3 If $H_{\mathrm{I}}$ is symmetric, then $E(\lambda)$ is real. In this case, if $E(\lambda)=\varepsilon_{0}(\lambda)$, Corollary 2.10 gives a sufficient condition for (H.5) to hold (see also Remark 2.11).

Under assumptions (H.2) and (H.5), $E(\lambda)$ is in $\rho\left(H_{0}\right)$. Hence $H_{0}-E(\lambda)$ is bijective with bounded inverse $\left(H_{0}-E(\lambda)\right)^{-1}$.

Lemma 3.4 Assume (H.1)-(H.5). Then, for all $\Xi \in D\left(\left(H_{0}^{\prime}-E_{0}\right)^{-1 / 2}\right)$,

$$
\lim _{\lambda \rightarrow 0}\left\langle\Xi,\left(H_{0}-E(\lambda)\right)^{-1} \Xi\right\rangle=\left\|\left(H_{0}^{\prime}-E_{0}\right)^{-1 / 2} \Xi\right\|^{2} .
$$

Proof. We denote by $E_{H_{0}}$ the spectral measure of $H_{0}$. Since $\Xi$ is in $\mathcal{H}_{0}^{\perp}$, it follows that

$$
\left\langle\Xi,\left(H_{0}-E(\lambda)\right)^{-1} \Xi\right\rangle=\left\langle\Xi,\left(H_{0}^{\prime}-E(\lambda)\right)^{-1} \Xi\right\rangle=\int_{\left(E_{0}, \infty\right)} \frac{1}{\mu-E(\lambda)} d\left\|E_{H_{0}}(\mu) \Xi\right\|^{2} .
$$

We have

$$
0<\frac{1}{\mu-E(\lambda)}<\frac{1}{\mu-E_{0}}, \quad \forall \mu>E_{0}
$$

and $\int_{\left(E_{0}, \infty\right)} 1 /\left(\mu-E_{0}\right) d\left\|E_{H_{0}}(\mu) \Xi\right\|^{2}<\infty$, because $\Xi$ is in $D\left(\left(H_{0}^{\prime}-E_{0}\right)^{-1 / 2}\right)$. By (3.8), $\lim _{\lambda \rightarrow 0} 1 /(\mu-E(\lambda))=1 /\left(\mu-E_{0}\right)$ for all $\mu>E_{0}$. Hence, by the Lebesgue dominated convergence theorem, we obtain

$$
\lim _{\lambda \rightarrow 0}\left\langle\Xi,\left(H_{0}-E(\lambda)\right)^{-1} \Xi\right\rangle=\int_{\left(E_{0}, \infty\right)} \frac{1}{\mu-E_{0}} d\left\|E_{H_{0}}(\mu) \Xi\right\|^{2}=\left\|\left(H_{0}^{\prime}-E_{0}\right)^{-1 / 2} \Xi\right\|^{2} .
$$


For $\lambda \in \mathbb{I}_{r}^{\times}$, we define operators $K(\lambda)$ and $G(\lambda)$ by

$$
\begin{aligned}
& K(\lambda):=\left(E(\lambda)-H_{0}\right)^{-1} Q_{0} H_{\mathrm{I}}, \\
& G(\lambda):=H_{\mathrm{I}}\left(E(\lambda)-H_{0}\right)^{-1} Q_{0} .
\end{aligned}
$$

It follows that

$$
K(\lambda)^{*} \supset G(\lambda)
$$

where, for a densely defined linear operator $A$ on a Hilbert space, $A^{*}$ denotes the adjoint of $A$.

The next theorem gives the second order asymptotics of $E(\lambda)$ in $\lambda$ near 0 :

Theorem 3.5 Assume (H.1)-(H.5). Suppose that

$$
\Psi_{0} \in D\left(G(\lambda) H_{\mathrm{I}}\right) \cap D\left(\left(H_{0}^{\prime}-E_{0}\right)^{-1 / 2} Q_{0} H_{\mathrm{I}}\right)
$$

for all $\lambda \in \mathbb{I}_{r}^{\times}$with

$$
\sup _{\lambda \in \mathbb{I}_{r}^{\times}}\left\|G(\lambda) H_{\mathrm{I}} \Psi_{0}\right\|<\infty
$$

Then

$$
E(\lambda)=E_{0}+\lambda\left\langle\Psi_{0}, H_{\mathrm{I}} \Psi_{0}\right\rangle-\lambda^{2}\left\|\left(H_{0}^{\prime}-E_{0}\right)^{-1 / 2} Q_{0} H_{\mathrm{I}} \Psi_{0}\right\|^{2}+o\left(\lambda^{2}\right) \quad(\lambda \rightarrow 0) .
$$

Proof. We have by (3.9), (3.3) and (3.2)

$$
\begin{aligned}
E(\lambda)= & E_{0}+\lambda\left\langle\Psi_{0}, H_{\mathrm{I}} \Psi_{0}\right\rangle-\lambda^{2}\left\|\left(H_{0}^{\prime}-E_{0}\right)^{-1 / 2} Q_{0} H_{\mathrm{I}} \Psi_{0}\right\|^{2} \\
& +\lambda^{2} R_{\lambda}+\lambda^{2}\left\langle G(\lambda) H_{\mathrm{I}} \Psi_{0}, \Phi(\lambda)\right\rangle
\end{aligned}
$$

where

$$
R_{\lambda}:=\left\langle H_{\mathrm{I}} \Psi_{0}, K(\lambda) \Psi_{0}\right\rangle+\left\|\left(H_{0}^{\prime}-E_{0}\right)^{-1 / 2} Q_{0} H_{\mathrm{I}} \Psi_{0}\right\|^{2}
$$

Hence

$$
\begin{aligned}
& \frac{E(\lambda)-E_{0}-\lambda\left\langle\Psi_{0}, H_{\mathrm{I}} \Psi_{0}\right\rangle+\lambda^{2}\left\|\left(H_{0}^{\prime}-E_{0}\right)^{-1 / 2} Q_{0} H_{\mathrm{I}} \Psi_{0}\right\|^{2}}{\lambda^{2}} \\
& =R_{\lambda}+\left\langle G(\lambda) H_{\mathrm{I}} \Psi_{0}, \Phi(\lambda)\right\rangle .
\end{aligned}
$$

By Lemma 3.4, $\lim _{\lambda \rightarrow 0} R_{\lambda}=0$. We have by the Schwarz inequality, (3.14) and (3.5)

$$
\left|\left\langle G(\lambda) H_{\mathrm{I}} \Psi_{0}, \Phi(\lambda)\right\rangle\right| \leq\left(\sup _{\lambda^{\prime} \in \mathbb{I}_{r}^{\times}}\left\|G\left(\lambda^{\prime}\right) H_{\mathrm{I}} \Psi_{0}\right\|\right)\|\Phi(\lambda)\| \rightarrow 0(\lambda \rightarrow 0) .
$$

Thus (3.15) holds. 


\section{Asymptotic Expansions up to Arbitrary Finite Or- ders in $\lambda$}

In this section, under assumptions (H.1)-(H.5), we derive an asymptotic expansion of $E(\lambda)$ up to an arbitrary finite order in $\lambda$. Let

$$
K_{0}:=\left(E_{0}-H_{0}^{\prime}\right)^{-1} Q_{0} H_{\mathrm{I}}
$$

For each $\ell \in \mathbb{N}$, we define an operator-valued function $K_{\ell}$ on $\mathbb{R}^{\ell}$ by

$$
\begin{array}{r}
K_{\ell}\left(x_{1}, \ldots, x_{\ell}\right):=\sum_{r=1}^{\ell}(-1)^{r} \sum_{\substack{j_{1}+\cdots+j_{r}=\ell \\
j_{1}, \ldots, j_{r} \geq 1}} x_{j_{1}} \cdots x_{j_{r}}\left(E_{0}-H_{0}^{\prime}\right)^{-(r+1)} Q_{0} H_{\mathrm{I}}, \\
\quad\left(x_{1}, \ldots, x_{\ell}\right) \in \mathbb{R}^{\ell} .
\end{array}
$$

For a natural number $N \geq 2$, we define a sequence $\left\{a_{n}\right\}_{n=1}^{N}$ as follows:

$$
\begin{aligned}
& a_{1}:=\left\langle\Psi_{0}, H_{\mathrm{I}} \Psi_{0}\right\rangle, \\
& a_{n}=\sum_{\substack{q+\ell=n \\
q, \ell \geq 1}} \sum_{\begin{array}{r}
\ell_{1}+\ldots+\ell_{q}=\ell-1 \\
\ell_{1}, \ldots, \ell_{q} \geq 0
\end{array}}\left\langle H_{\mathrm{I}} \Psi_{0}, K_{l_{1}}\left(a_{1}, \ldots, a_{\ell_{1}}\right) \cdots K_{l_{q}}\left(a_{1}, \ldots, a_{\ell_{q}}\right) \Psi_{0}\right\rangle, \\
& n=2, \ldots, N
\end{aligned}
$$

provided that

$$
\Psi_{0} \in \cap_{n=2}^{N} \bigcap_{\substack{q+\ell=n \\ q, \ell \geq 1}}^{\bigcap_{\substack{\ell_{1}+\cdots+\ell_{q}=\ell-1 \\ \ell_{1}, \ldots, \ell_{q} \geq 0}} \cap_{r_{1}=0}^{\ell_{1}} \cdots \cap_{r_{q}=0}^{\ell_{q}} D} D\left(\prod_{j=1}^{q}\left(E_{0}-H_{0}^{\prime}\right)^{-\left(r_{j}+1\right)} Q_{0} H_{\mathrm{I}}\right),
$$

where, for (not necessarily commuting) linear operators $A_{1}, \ldots, A_{q}$ on a Hilbert space, $\prod_{j=1}^{q} A_{j}:=A_{1} A_{2} \cdots A_{q}$.

Note that, on the right hand side of $(4.4)$, only $a_{j}(j=1, \ldots, n-2)$ appears. Hence $a_{n}$ 's with $n \geq 3$ are uniquely determined.

We have

$$
\begin{aligned}
& a_{2}=-\left\langle H_{\mathrm{I}} \Psi_{0},\left(H_{0}^{\prime}-E_{0}\right)^{-1} Q_{0} H_{\mathrm{I}} \Psi_{0}\right\rangle \leq 0, \\
& \begin{aligned}
& a_{3}=\left\langle\left(H_{0}^{\prime}-E_{0}\right)^{-1} H_{\mathrm{I}} \Psi_{0}, H_{\mathrm{I}}\left(H_{0}^{\prime}-E_{0}\right)^{-1} Q_{0} H_{\mathrm{I}} \Psi_{0}\right\rangle \\
& \quad-\left\langle\Psi_{0}, H_{\mathrm{I}} \Psi_{0}\right\rangle\left\|\left(H_{0}^{\prime}-E_{0}\right)^{-1} Q_{0} H_{\mathrm{I}} \Psi_{0}\right\|^{2} .
\end{aligned}
\end{aligned}
$$

Theorem 4.1 Let $N \geq 2$ be a natural number. Assume (H.1)-(H.5). Suppose that (4.5) holds and $\Psi_{0} \in \cap_{n=1}^{N-1} D\left(G(\lambda)^{n} H_{\mathrm{I}}\right)$ with

$$
\sup _{r \in \mathbb{I}_{r}^{\times}}\left\|G(\lambda)^{n} H_{\mathrm{I}} \Psi_{0}\right\|<\infty, \quad n=1, \ldots, N-1 .
$$


Then

$$
E(\lambda)=E_{0}+\sum_{n=1}^{N} a_{n} \lambda^{n}+o\left(\lambda^{N}\right) \quad(\lambda \rightarrow 0)
$$

Proof. We prove (4.9) by induction in $N \geq 2$. Eq. (4.9) with $N=2$ follows from Theorem 3.5.

Suppose that (4.9) holds with some $N \geq 2$. Let

$$
K_{r}(\lambda):=\left(E_{0}-E(\lambda)\right)^{r}\left(E_{0}-H_{0}^{\prime}\right)^{-(r+1)} Q_{0} H_{\mathrm{I}}, \quad r=0,1, \ldots, N-1,
$$

and $K(\lambda)$ be as in (3.11). Then, using the easily proven formula

$$
\left(E(\lambda)-H_{0}\right)^{-1} Q_{0}=\left(E_{0}-H_{0}^{\prime}\right)^{-1} Q_{0}+\left(E_{0}-E(\lambda)\right)\left(E(\lambda)-H_{0}\right)^{-1}\left(E_{0}-H_{0}^{\prime}\right)^{-1} Q_{0},
$$

we obtain

$$
K(\lambda)=\sum_{r=0}^{N-1} K_{r}(\lambda)+\left(E_{0}-E(\lambda)\right)^{N}\left(E(\lambda)-H_{0}\right)^{-1}\left(E_{0}-H_{0}^{\prime}\right)^{-N} Q_{0} H_{\mathrm{I}},
$$

on $\cap_{r=1}^{N} D\left(\left(E_{0}-H_{0}^{\prime}\right)^{-r} Q_{0} H_{\mathrm{I}}\right)$. By $($ H.5 $)$, for all $\Psi \in D\left(\left(E_{0}-H_{0}^{\prime}\right)^{-(N+1)} Q_{0} H_{\mathrm{I}}\right)$, we have

$$
\left\|\left(E(\lambda)-H_{0}\right)^{-1}\left(E_{0}-H_{0}^{\prime}\right)^{-N} Q_{0} H_{\mathrm{I}} \Psi\right\| \leq\left\|\left(E_{0}-H_{0}^{\prime}\right)^{-(N+1)} Q_{0} H_{\mathrm{I}} \Psi\right\| .
$$

Hence, by (3.7), we obtain

$$
\lim _{\lambda \rightarrow 0} \frac{\left(E_{0}-E(\lambda)\right)^{N}\left(E(\lambda)-H_{0}\right)^{-1}\left(E_{0}-H_{0}^{\prime}\right)^{-N} Q_{0} H_{\mathrm{I}} \Psi}{\lambda^{N-1}}=0,
$$

i.e.

$$
\left(E_{0}-E(\lambda)\right)^{N}\left(E(\lambda)-H_{0}\right)^{-1}\left(E_{0}-H_{0}^{\prime}\right)^{-N} Q_{0} H_{\mathrm{I}} \Psi=o\left(\lambda^{N-1}\right) \quad(\lambda \rightarrow 0) .
$$

By the induction hypothesis (4.9), we have

$$
E_{0}-E(\lambda)=-\sum_{n=1}^{N} a_{n} \lambda^{n}+o\left(\lambda^{N}\right) \quad(\lambda \rightarrow 0)
$$

Hence

$$
K_{r}(\lambda)=(-1)^{r} \sum_{j_{1}, \ldots, j_{r}=1}^{N} a_{j_{1}} \cdots a_{j_{r}} \lambda^{j_{1}+\cdots+j_{r}}\left(E_{0}-H_{0}^{\prime}\right)^{-(r+1)} Q_{0} H_{\mathrm{I}}+o\left(\lambda^{N+1}\right) \quad(\lambda \rightarrow 0)
$$

on $D\left(\left(E_{0}-H_{0}^{\prime}\right)^{-(r+1)} Q_{0} H_{\mathrm{I}}\right)$. Putting this into $K_{r}(\lambda)$ on the right hand side of $(4.10)$, we obtain

$$
K(\lambda)=K_{0}+\sum_{\ell=1}^{N-1} K_{\ell}\left(a_{1}, \ldots, a_{\ell}\right) \lambda^{\ell}+o\left(\lambda^{N-1}\right) \quad(\lambda \rightarrow 0)
$$


on $\cap_{r=1}^{N+1} D\left(\left(E_{0}-H_{0}^{\prime}\right)^{-r} Q_{0} H_{\mathrm{I}}\right)$, where $K_{\ell}$ is defined by $(4.2)$.

On the other hand, we have by (3.9) and (3.3)

$$
E(\lambda)=E_{0}+\left\langle\Psi_{0}, H_{\mathrm{I}} \Psi_{0}\right\rangle \lambda+\sum_{k=1}^{N}\left\langle H_{\mathrm{I}} \Psi_{0}, K(\lambda)^{k} \Psi_{0}\right\rangle \lambda^{k+1}+\left\langle H_{\mathrm{I}} \Psi_{0}, K(\lambda)^{N} \Phi(\lambda)\right\rangle \lambda^{N+1} .
$$

Using (4.11), we have

$$
\begin{aligned}
E(\lambda)= & E_{0}+\left\langle\Psi_{0}, H_{\mathrm{I}} \Psi_{0}\right\rangle \lambda \\
& +\sum_{k=1}^{N} \sum_{\ell_{1}, \ldots, \ell_{k}=0}^{N-1}\left\langle H_{\mathrm{I}} \Psi_{0}, K_{\ell_{1}}\left(a_{1}, \ldots, a_{\ell_{1}}\right) \cdots K_{\ell_{k}}\left(a_{1}, \ldots, a_{\ell_{k}}\right) \Psi_{0}\right\rangle \\
& \times \lambda^{\ell_{1}+\cdots+\ell_{k}+k+1}+o\left(\lambda^{N+1}\right)+\left\langle H_{\mathrm{I}} \Psi_{0}, K(\lambda)^{N} \Phi(\lambda)\right\rangle \lambda^{N+1} \quad(\lambda \rightarrow 0) .
\end{aligned}
$$

By rearranging the sums on $k$ and $\ell_{1}, \ldots, \ell_{k}$, we obtain

$$
\begin{aligned}
E(\lambda)= & E_{0}+\left\langle\Psi_{0}, H_{\mathrm{I}} \Psi_{0}\right\rangle \lambda \\
& +\sum_{n=2}^{N+1} a_{n} \lambda^{n}+o\left(\lambda^{N+1}\right)+\left\langle H_{\mathrm{I}} \Psi_{0}, K(\lambda)^{N} \Phi(\lambda)\right\rangle \lambda^{N+1} \quad(\lambda \rightarrow 0) .
\end{aligned}
$$

By condition (4.8) with $N-1$ replaced by $N$, we have

$$
\left|\left\langle H_{\mathrm{I}} \Psi_{0}, K(\lambda)^{N} \Phi(\lambda)\right\rangle\right| \leq\left(\sup _{\lambda^{\prime} \in \mathbb{I}_{r}^{\times}}\left\|G\left(\lambda^{\prime}\right)^{N} H_{\mathrm{I}} \Psi_{0}\right\|\right)\|\Phi(\lambda)\| \rightarrow 0 \quad(\lambda \rightarrow 0) .
$$

Hence

$$
\left\langle H_{\mathrm{I}} \Psi_{0}, K(\lambda)^{N} \Phi(\lambda)\right\rangle \lambda^{N+1}=o\left(\lambda^{N+1}\right) \quad(\lambda \rightarrow 0) .
$$

Thus (4.9) holds with $N$ replaced by $N+1$.

\section{Application to the GSB Model}

In this section, we apply Theorem 4.1 to the GSB model mentioned in Introduction. Following [5], we first review the GSB model briefly .

The quantum filed in the GSB model is a scalar Bose field acting in the boson (symmetric) Fock space

$$
\mathcal{F}_{\mathrm{b}}\left(L^{2}\left(\mathbb{R}^{\nu}\right)\right):=\oplus_{n=0}^{\infty}\left[\otimes_{\mathrm{s}}^{n} L^{2}\left(\mathbb{R}^{\nu}\right)\right]
$$

over the Hilbert space $L^{2}\left(\mathbb{R}^{\nu}\right)(\nu \in \mathbb{N})$, where $\otimes_{\mathrm{s}}^{n} L^{2}\left(\mathbb{R}^{\nu}\right)$ denotes the $n$-fold symmetric tensor product of $L^{2}\left(\mathbb{R}^{\nu}\right)$ with convention $\otimes_{\mathrm{s}}^{0} L^{2}\left(\mathbb{R}^{\nu}\right):=\mathbb{C}$.

We denote by $a(f)\left(f \in L^{2}\left(\mathbb{R}^{\nu}\right)\right)$ the annihilation operator with test function $f$, i.e., it is a densely defined closed linear operator on $\mathcal{F}_{\mathrm{b}}\left(L^{2}\left(\mathbb{R}^{\nu}\right)\right)$ such that its adjoint $a(f)^{*}$ 
obeys the following properties: For all $\psi \in D\left(a(f)^{*}\right),\left(a(f)^{*} \psi\right)^{(0)}=0$ and $\left(a(f)^{*} \psi\right)^{(n)}=$ $\sqrt{n} S_{n}\left(f \otimes \psi^{(n-1)}\right), n \geq 1$, where $S_{n}$ is the symmetrization operator on the $n$-fold tensor product $\otimes^{n} L^{2}\left(\mathbb{R}^{\nu}\right)$ of $L^{2}\left(\mathbb{R}^{\nu}\right)$. The time-zero field for the GSB model is defined by

$$
\phi(f):=\frac{1}{2}\left(a(f)^{*}+a(f)\right),
$$

which is called the Segal field operator with test function $f$.

The one-particle Hamiltonian of a free boson, which acts in $L^{2}\left(\mathbb{R}^{\nu}\right)$, is described by the multiplication operator of an energy function $\omega: \mathbb{R}^{\nu} \rightarrow[0, \infty) ; \mathbb{R}^{\nu} \ni k \mapsto \omega(k) \geq 0$ which is Borel measurable and $0<\omega(k)<\infty$ for almost everywhere (a.e.) $k \in \mathbb{R}^{\nu}$. We denote the one-particle Hamiltonian by the same symbol $\omega$. Then the Hamiltonian of the free quantum field with one-particle Hamiltonian $\omega$ is defined by

$$
H_{\mathrm{b}}:=\oplus_{n=0}^{\infty} \omega^{(n)}
$$

the second quantization of $\omega$, where $\omega^{(0)}:=0$ as an operator on $\mathbb{C}, \omega^{(1)}:=\omega$ and, for $n \geq 2, \omega^{(n)}:=\sum_{j=1}^{n} I \otimes \cdots \otimes \stackrel{j \text { th }}{\omega} \otimes \cdots \otimes I$ acting in $\otimes_{\mathrm{s}}^{n} L^{2}\left(\mathbb{R}^{\nu}\right)(I$ denotes the identity on $\left.L^{2}\left(\mathbb{R}^{\nu}\right)\right)$.

For more detailed descriptions on the boson Fock space theory, we refer the reader to $[17, \S \mathrm{X} .7]$.

Let

$$
\omega_{0}:=\operatorname{ess}_{\operatorname{minf}} \operatorname{li}^{\nu} \omega(k) \geq 0 .
$$

If $\omega_{0}>0$ (resp. $\omega_{0}=0$ ), then we say that the Bose field or the boson is massive (resp. massless) $)^{2}$.

We take the Hilbert space of quantum particles interacting with the quantum scalar filed to be an abstract complex Hilbert space $\mathcal{K}$. Then a Hilbert space of state vectors for the GSB model is given by

$$
\mathcal{H}_{\mathrm{GSB}}:=\mathcal{K} \otimes \mathcal{F}_{\mathrm{b}}\left(L^{2}\left(\mathbb{R}^{\nu}\right)\right)
$$

Let $L_{\text {sym }}^{2}\left(\left(\mathbb{R}^{\nu}\right)^{n} ; \mathcal{K}\right)$ be the Hilbert space of $\mathcal{K}$-valued symmetric $L^{2}$-functions on $\left(\mathbb{R}^{\nu}\right)^{n}$ with convention $L_{\text {sym }}^{2}\left(\left(\mathbb{R}^{\nu}\right)^{0} ; \mathcal{K}\right):=\mathcal{K}$. Then the Hilbert space $\mathcal{H}_{\mathrm{GSB}}$ has a natural identification as

$$
\begin{aligned}
\mathcal{H}_{\mathrm{GSB}} & =\oplus_{n=0}^{\infty} L_{\text {sym }}^{2}\left(\left(\mathbb{R}^{\nu}\right)^{n} ; \mathcal{K}\right) \\
& =\left\{\Psi=\left\{\Psi^{(n)}\right\}_{n=0}^{\infty} \mid \Psi^{(n)} \in L_{\text {sym }}^{2}\left(\left(\mathbb{R}^{\nu}\right)^{n} ; \mathcal{K}\right), n \geq 0, \sum_{n=0}^{\infty}\left\|\Psi^{(n)}\right\|^{2}<\infty\right\} .
\end{aligned}
$$

${ }^{2}$ An example of $\omega$ for a relativistic Bose field is given by $\omega(k)=\sqrt{k^{2}+m^{2}}$ with $m \geq 0$ being the mass of the boson. In this case, we have $\omega_{0}=m$, showing that $\omega_{0}$ is certainly the mass of the boson. But, in general (mathematically), $\omega_{0}$ is not necessarily a mass in the physical sense. For example, if the boson is non-relativistic, then one may take $\omega(k)=k^{2} / 2 m$. In this case, $\omega_{0}(=0)$ is not a mass. 
For each $n \geq 0$, we call the subspace

$$
\mathcal{H}^{(n)}:=\left\{\Psi=\left\{0, \ldots, 0, \Psi^{(n)}, 0, \ldots\right\} \in \mathcal{H}_{\mathrm{GSB}} \mid \Psi^{(n)} \in L_{\mathrm{sym}}^{2}\left(\left(\mathbb{R}^{\nu}\right)^{n} ; \mathcal{K}\right)\right\}
$$

the $n$-boson space of the GSB model. We have

$$
\mathcal{H}_{\mathrm{GSB}}=\oplus_{n=0}^{\infty} \mathcal{H}^{(n)}
$$

We assume that the Hamiltonian of the system of quantum particles is given by a self-adjoint operator $A$ on $\mathcal{K}$ which is bounded below. Then

$$
E_{0}:=\inf \sigma(A)
$$

is finite and

$$
\widetilde{A}:=A-E_{0}
$$

is a non-negative self-adjoint operator on $\mathcal{K}$.

Let $J \in \mathbb{N}$ and $B_{j}(j=1, \ldots, J)$ be a symmetric operator on $\mathcal{K}$. Let $g_{j} \in L^{2}\left(\mathbb{R}^{\nu}\right)$ $(j=1, \ldots, J)$ and $\lambda \in \mathbb{R}$. In this section, we take $H_{0}$ and $H_{\mathrm{I}}$ as follows:

$$
\begin{aligned}
& H_{0}:=A \otimes I+I \otimes H_{\mathrm{b}}, \\
& H_{\mathrm{I}}:=\sum_{j=1}^{J} B_{j} \otimes \phi\left(g_{j}\right) .
\end{aligned}
$$

The function $g_{j}$ in $\phi\left(g_{j}\right)$ physically means a momentum cutoff function of the boson. The Hamiltonian of the GSB model is defined by

$$
H_{\mathrm{GSB}}(\lambda):=H_{0}+\lambda H_{\mathrm{I}}
$$

Before going into detailed analysis on the GSB model, we take this opportunity to report an important fact on the lowest (minimal) energy of $H_{\mathrm{GSB}}(\lambda)$ in the case where $H_{\mathrm{GSB}}(\lambda)$ is self-adjoint and bounded below. Hence, let

$$
\Lambda:=\left\{\lambda \in \mathbb{R} \mid H_{\mathrm{GSB}}(\lambda) \text { is self-adjoint and bounded below }\right\}
$$

and, for each $\lambda \in \Lambda$,

$$
E_{0}(\lambda):=\inf \sigma\left(H_{\mathrm{GSB}}(\lambda)\right)
$$

Theorem 5.1 The set $\Lambda$ is reflection symmetric with respect to the origin of $\mathbb{R}$ (i.e., $\lambda \in \Lambda \Longleftrightarrow-\lambda \in \Lambda$ ) and $E_{0}(\cdot)$ is an even function on $\Lambda$ :

$$
E_{0}(\lambda)=E_{0}(-\lambda), \quad \lambda \in \Lambda .
$$


Proof. Let $\lambda \in \Lambda$. We denote by $N_{\mathrm{b}}$ the number operator on $\mathcal{F}_{\mathrm{b}}\left(L^{2}\left(\mathbb{R}^{\nu}\right)\right):\left(N_{\mathrm{b}} \Psi\right)^{(n)}=$ $n \Psi^{(n)}, n \geq 0, \Psi \in D(N)$. The operator $N_{\mathrm{b}}$ is self-adjoint and non-negative. Hence $U:=e^{i \pi N_{\mathrm{b}}}$ is unitary. We have the operator equality

$$
U a(f)^{\#} U^{-1}=-a(f)^{\#}, \quad f \in L^{2}\left(\mathbb{R}^{\nu}\right),
$$

where $a(f)^{\#}$ denotes either $a(f)$ or $a(f)^{*}$. Hence

$$
U \phi(f) U^{-1}=-\phi(f), \quad f \in L^{2}\left(\mathbb{R}^{\nu}\right) .
$$

Also we have

$$
U H_{\mathrm{b}} U^{-1}=H_{\mathrm{b}}
$$

Hence we obtain

$$
(I \otimes U) H_{\mathrm{GSB}}(\lambda)(I \otimes U)^{-1}=H_{\mathrm{GSB}}(-\lambda),
$$

which implies that $\Lambda$ is reflection symmetric with respect to the origin of $\mathbb{R}$ and

$$
\sigma\left(H_{\mathrm{GSB}}(\lambda)\right)=\sigma\left(H_{\mathrm{GSB}}(-\lambda)\right)
$$

Hence (5.11) holds.

Remark 5.2 The proof given above can be applied also to a general particle-field Hamiltonian [4], which includes Pauli-Fierz type models (e.g., [8, 9, 14]) and Nelson type models (e.g., $[2,16])$, and the Dereziński-Gérard model [11] to conclude that their minimal energy is an even function of the coupling constant.

In what follows, we assume the following conditions:

(A.1) The operator $A$ has compact resolvent.

(A.2) Each $B_{j}(j=1, \ldots, J)$ is $\widetilde{A}^{1 / 2}$-bounded. Namely, $D\left(\widetilde{A}^{1 / 2}\right) \subset \cap_{j=1}^{J} D\left(B_{j}\right)$ and there exist constants $a_{j} \geq 0, b_{j} \geq 0$ such that, for all $\psi \in D\left(\widetilde{A}^{1 / 2}\right)$,

$$
\left\|B_{j} \psi\right\| \leq a_{j}\left\|\widetilde{A}^{1 / 2} \psi\right\|+b_{j}\|\psi\|, \quad j=1, \ldots, J .
$$

(A.3) $g_{j}, g_{j} / \omega \in L^{2}\left(\mathbb{R}^{\nu}\right), j=1, \ldots, J$.

(A.4) The function $\omega$ is continuous on $\mathbb{R}^{\nu}$ with $\lim _{|k| \rightarrow \infty} \omega(k)=\infty$ and there exist constants $\gamma>0$ and $C>0$ such that

$$
\left|\omega(k)-\omega\left(k^{\prime}\right)\right| \leq C\left|k-k^{\prime}\right|^{\gamma}\left(1+\omega(k)+\omega\left(k^{\prime}\right)\right), \quad k, k^{\prime} \in \mathbb{R}^{\nu} .
$$


Remark 5.3 Under (A.1), $A$ has purely discrete spectrum $\left\{E_{n}\right\}_{n=0}^{N}$ with $E_{0}<E_{1}<$ $\cdots<E_{n}<E_{n+1}<\cdots$, where $N<\infty$ or $N=\infty$. In particular, $E_{0}$ is an eigenvalue of $A$ with a finite multiplicity. Under assumption (A.4), we have $\sigma(\omega)=\left[\omega_{0}, \infty\right)$, where $\omega_{0}$ is given by (5.1). Hence it follows that

$$
\sigma\left(H_{0}\right)=\left\{E_{0}, \ldots, E_{n_{0}}\right\} \cup\left[E_{0}+\omega_{0}, \infty\right)
$$

where $n_{0}:=\max \left\{n \geq 0 \mid E_{n}<E_{0}+\omega_{0}\right\}$. In particular, if the boson is massless, then $\sigma\left(H_{0}\right)=\left[E_{0}, \infty\right)$, which shows that all the eigenvalues of $H_{0}$ are embedded eigenvalues of $H_{0}$. Hence, in this case, for each $E_{n}$, one can neither apply the standard analytic perturbation theory nor the standard asymptotic perturbation theory. Thus we need a new approach in the massless case. Note that, in the massive case $\omega_{0}>0, E_{n}(n=$ $\left.0,1, \ldots, n_{0}\right)$ is an isolated eigenvalue of $H_{0}$. We can show that $H_{\mathrm{I}}$ is $H_{0}$-bounded [5]. Hence, in the massive case, we can apply the standard analytic perturbation theory to conclude that, for all sufficiently small $|\lambda|$ (its smallness may depend on $\omega_{0}$ ), $H_{\mathrm{GSB}}(\lambda)$ has a ground state and the ground state energy is analytic in $\lambda$.

Remark 5.4 As for the spectrum of $H_{\mathrm{GSB}}(\lambda)$, the following result is known [3, Theorem 3.3]: If (A.2) holds and $g_{j}, g_{j} / \sqrt{\omega} \in L^{2}\left(\mathbb{R}^{\nu}\right)(j=1, \ldots, J)$ with $\sigma(\omega)=[0, \infty)$, then, for all $\lambda \in \Lambda$,

$$
\sigma\left(H_{\mathrm{GSB}}(\lambda)\right)=\left[E_{0}(\lambda), \infty\right)
$$

Note that, for this fact, $H_{\mathrm{GSB}}(\lambda)$ does not necessarily have a ground state.

Let

$$
\Omega_{0}:=\{1,0,0, \ldots\} \in \mathcal{F}_{\mathrm{b}}\left(L^{2}\left(\mathbb{R}^{\nu}\right)\right)
$$

be the Fock vacuum and $P_{\Omega_{0}}$ be the orthogonal projections onto the one-dimensional subspace $\left\{\alpha \Omega_{0} \mid \alpha \in \mathbb{C}\right\}$. We denote by $p_{0}$ the orthogonal projection onto ker $\widetilde{A}$.

As for the existence of a ground state of $H_{\mathrm{GSB}}(\lambda)$, we have the following theorem [5]:

Theorem 5.5 Assume (A.1)-(A.4). Then there exists a constant $r>0$ independent of $\lambda$ such that the following hold:

(i) $(-r, r) \subset \Lambda$.

(ii) For all $\lambda \in(-r, r), H_{\mathrm{GSB}}(\lambda)$ has a ground state $\Psi_{0}(\lambda)$ and there exists a constant $M>0$ independent of $\lambda \in(-r, r)$ such that, for all $|\lambda|<r,\left\|\Psi_{0}(\lambda)\right\| \leq 1$ and

$$
\left\langle\Psi_{0}(\lambda), p_{0} \otimes P_{\Omega_{0}} \Psi_{0}(\lambda)\right\rangle \geq 1-\lambda^{2} M^{2}>0
$$


Under the assumption of Theorem $5.5, E_{0}(\lambda)$ with $\lambda \in(-r, r)$ is the ground state energy of $H_{\mathrm{GSB}}(\lambda)$. To derive asymptotic expansions for $E_{0}(\lambda)$ in $\lambda$ near 0 , we need to check that the assumptions taken in Sections 3 and 4 hold in the case where $H(\lambda)=$ $H_{\mathrm{GSB}}(\lambda)$. For this purpose, however, we need additional assumptions:

(A.5) The eigenvalue of $E_{0}$ of $A$ is simple and there exists a $j_{0} \in\{1, \ldots, J\}$ such that $B_{j_{0}} \psi \neq 0$ for all $\psi \in \operatorname{ker} \widetilde{A} \backslash\{0\}$.

(A.6) The set $\left\{g_{1}, \ldots, g_{J}\right\} \subset L^{2}\left(\mathbb{R}^{\nu}\right)$ is linearly independent.

We denote by $\psi_{0}$ a normalized eigenvector of $A$ with eigenvalue $E_{0}$ :

$$
A \psi_{0}=E_{0} \psi_{0}, \quad\left\|\psi_{0}\right\|=1
$$

Hence ker $\widetilde{A}=\left\{\alpha \psi_{0} \mid \alpha \in \mathbb{C}\right\}$. By (A.5), we have

$$
B_{j_{0}} \psi_{0} \neq 0
$$

It follows from the theory of tensor products of self-adjoint operators that $E_{0}$ is a simple eigenvalue of $H_{0}$ being the ground state energy of $H_{0}$ with a normalized eigenvector

$$
\Psi_{0}:=\psi_{0} \otimes \Omega_{0}
$$

By the well kown fact that

$$
a(f) \Omega_{0}=0, \quad f \in L^{2}\left(\mathbb{R}^{\nu}\right)
$$

we have

$$
\left\langle\Psi_{0}, H_{\mathrm{I}} \Psi_{0}\right\rangle=0
$$

Hence (2.19) holds.

In the present model, the orthogonal projection $P_{0}$ onto $\operatorname{ker}\left(H_{0}-E_{0}\right)$ takes the form

$$
P_{0}=p_{0} \otimes P_{\Omega_{0}}
$$

Hence

$$
Q_{0}=I-P_{0}=I-p_{0} \otimes P_{\Omega_{0}}
$$

Lemma 5.6 Assume (A.1)-(A.6). Then $Q_{0} H_{\mathrm{I}} \Psi_{0} \neq 0$.

Proof. By (5.18), we have

$$
H_{\mathrm{I}} \Psi_{0}=\sum_{j=1}^{J} \frac{1}{\sqrt{2}} B_{j} \psi_{0} \otimes a\left(g_{j}\right)^{*} \Omega_{0} .
$$


Since $P_{\Omega_{0}} a(f)^{*} \Omega_{0}=0$ for all $f \in L^{2}\left(\mathbb{R}^{\nu}\right)$, we have

$$
Q_{0} H_{\mathrm{I}} \Psi_{0}=\sum_{j=1}^{J} \frac{1}{\sqrt{2}} B_{j} \psi_{0} \otimes a\left(g_{j}\right)^{*} \Omega_{0}
$$

Suppose that $Q_{0} H_{\mathrm{I}} \Psi_{0}$ were a zero vector. Then it follows that, for all $\psi \in \mathcal{K}$,

$$
\sum_{j=1}^{J}\left\langle\psi, B_{j} \psi_{0}\right\rangle g_{j}=0
$$

By (A.6), this implies that, for all $\psi \in \mathcal{K},\left\langle\psi, B_{j} \psi_{0}\right\rangle=0, j=1, \ldots, J$. Hence $B_{j} \psi_{0}=0$ for all $j=1, \ldots, J$. But this contradicts (5.16).

As in the case of the general theory in Section 2, we denote by $H_{0}^{\prime}$ the reduced part of $H_{0}$ to $\left\{\alpha \Psi_{0} \mid \alpha \in \mathbb{C}\right\}^{\perp}$.

Let $H_{\mathrm{b}}^{\prime}$ be the reduced part of $H_{\mathrm{b}}$ to $\left\{\alpha \Omega_{0} \mid \alpha \in \mathbb{C}\right\}^{\perp}$. Then, in the GSB model, we have, under the identification (5.3),

$$
H_{0}^{\prime}-E_{0}=\widetilde{A}+H_{\mathrm{b}}^{\prime} .
$$

Lemma 5.7 Assume (A.1)-(A.5). Let $\psi \in \mathcal{K}$ and $f \in L^{2}\left(\mathbb{R}^{\nu}\right)$. Then $\psi \otimes a(f)^{*} \Omega_{0}$ is in $D\left(\left(H_{0}^{\prime}-E_{0}\right)^{-1}\right)$ if and only if

$$
\int_{\omega(k)>0}\left\|(\widetilde{A}+\omega(k))^{-1} \psi\right\|^{2}|f(k)|^{2} d k<\infty .
$$

In particular, if $f / \omega \in L^{2}\left(\mathbb{R}^{\nu}\right)$, then $\psi \otimes a(f)^{*} \Omega_{0} \in D\left(\left(H_{0}^{\prime}-E_{0}\right)^{-1}\right)$.

Proof. For a self-adjoint operator $S$, we denote by $E_{S}(\cdot)$ its spectral measure. We have $E_{H_{0}^{\prime}}\left(X_{1} \times X_{2}\right)=E_{A}\left(X_{1}\right) \otimes E_{H_{\mathrm{b}}^{\prime}}\left(X_{2}\right)$ for all Borel sets $X_{1}, X_{2} \subset \mathbb{R}$ and $E_{H_{\mathrm{b}}^{\prime}}(\{0\})=0$. Hence it follows that the vector $\psi \otimes a(f)^{*} \Omega_{0}$ is in $D\left(\left(H_{0}^{\prime}-E_{0}\right)^{-1}\right)$ if and only if

$$
I_{f}:=\int_{x+y>E_{0}} \frac{1}{\left|x-E_{0}+y\right|^{2}} d\left\|E_{A}(x) \psi\right\|^{2} d\left\|E_{H_{\mathrm{b}}^{\prime}}(y) a(f)^{*} \Omega_{0}\right\|^{2}<\infty
$$

We have

$$
\left(H_{\mathrm{b}}^{\prime} a(f)^{*} \Omega_{0}\right)^{(1)}(k)=\omega(k) f(k), \quad\left(H_{\mathrm{b}}^{\prime} a(f)^{*} \Omega_{0}\right)^{(n)}(k)=0, \quad n \neq 1 .
$$

Hence

$$
I_{f}=\int_{\omega(k)>0}\left\|(\widetilde{A}+\omega(k))^{-1} \psi\right\|^{2}|f(k)|^{2} d k .
$$

Thus the first statement of the present lemma holds.

Since $\widetilde{A} \geq 0$, we have $I_{f} \leq\|\psi\|^{2} \int_{\mathbb{R}^{\nu}}|f(k)|^{2} / \omega(k)^{2} d k$. Hecne, if $f / \omega \in L^{2}\left(\mathbb{R}^{\nu}\right)$, then $I_{f}<\infty$ and thus $\psi \otimes a(f)^{*} \Omega_{0} \in D\left(\left(H_{0}^{\prime}-E_{0}\right)^{-1}\right)$. 
Using the Schwarz inequality for the inner product and the elementary inequality

$$
\alpha \beta \leq \varepsilon^{2} \alpha^{2}+\frac{\beta^{2}}{4 \varepsilon^{2}} \quad(\alpha \geq 0, \beta \geq 0, \varepsilon>0),
$$

we have for all $\psi \in D(\widetilde{A})$ and all $\varepsilon>0$

$$
\left\|\widetilde{A}^{1 / 2} \psi\right\|^{2} \leq \varepsilon^{2}\|\widetilde{A} \psi\|^{2}+\frac{1}{4 \varepsilon^{2}}\|\psi\|^{2} .
$$

By this fact and (A.2), we obtain

$$
\left\|B_{j} \psi\right\| \leq a \varepsilon\|\widetilde{A} \psi\|+b_{\varepsilon}\|\psi\|, \quad \psi \in D(\widetilde{A}) \quad(j=1, \ldots, J),
$$

where $a:=\max \left\{a_{1}, \ldots, a_{J}\right\}, \varepsilon>0$ is arbitrary and

$$
b_{\varepsilon}:=b+\frac{a}{2 \varepsilon}
$$

with $b:=\max \left\{b_{1}, \ldots, b_{J}\right\}$.

Lemma 5.8 Assume (A.2). Then, for each $j=1, \ldots, J$ and $k \in \mathbb{R}^{\nu}$ with $\omega(k)>0$, $B_{j}(\widetilde{A}+\omega(k))^{-1}$ is bounded with

$$
\left\|B_{j}(\widetilde{A}+\omega(k))^{-1}\right\| \leq a \varepsilon+\frac{b_{\varepsilon}}{\omega(k)},
$$

where $\varepsilon>0$ is arbitrary.

Proof. By (5.24), we have for all $\psi \in \mathcal{K}$

$$
\left\|B_{j}(\widetilde{A}+\omega(k))^{-1} \psi\right\| \leq a \varepsilon\left\|\widetilde{A}(\widetilde{A}+\omega(k))^{-1} \psi\right\|+b_{\varepsilon}\left\|(\widetilde{A}+\omega(k))^{-1} \psi\right\| .
$$

We have $\left\|(\widetilde{A}+\omega(k))^{-1} \psi\right\| \leq \omega(k)^{-1}\|\psi\|$ and $\left\|\widetilde{A}(\widetilde{A}+\omega(k))^{-1} \psi\right\| \leq\|\psi\|$. Thus the desired result follows.

Lemma 5.9 Assume (A.1)-(A.6). Then (3.13) holds in the present model.

Proof. By (5.21) and the condition $g_{j} / \omega(j=1, \ldots, J)$, we can apply Lemma 5.7 to conclude that

$$
Q_{0} H_{\mathrm{I}} \Psi_{0} \in D\left(\left(H_{0}^{\prime}-E_{0}\right)^{-1}\right) .
$$

Using idetification (5.3), we have

$$
\begin{array}{ll}
\left(\left(H_{0}^{\prime}-E_{0}\right)^{-1} Q_{0} H_{\mathrm{I}} \Psi_{0}\right)^{(1)}(k)=\sum_{j=1}^{J} \frac{1}{\sqrt{2}} g_{j}(k)(\widetilde{A}+\omega(k))^{-1} B_{j} \psi_{0}, & \omega(k)>0, \\
\left(\left(H_{0}^{\prime}-E_{0}\right)^{-1} Q_{0} H_{\mathrm{I}} \Psi_{0}\right)^{(n)}=0, \quad n \neq 1 . & \omega
\end{array}
$$


Hence $\left(H_{0}^{\prime}-E_{0}\right)^{-1} Q_{0} H_{\mathrm{I}} \Psi_{0}$ is in $D\left(H_{\mathrm{I}}\right)$ with

$$
\begin{gathered}
\left(H_{\mathrm{I}}\left(H_{0}^{\prime}-E_{0}\right)^{-1} Q_{0} H_{\mathrm{I}} \Psi_{0}\right)^{(0)}=\frac{1}{2} \sum_{\ell, j=1}^{J} \int_{\omega(k)>0} d k g_{\ell}(k)^{*} g_{j}(k) B_{\ell}(\widetilde{A}+\omega(k))^{-1} B_{j} \psi_{0}, \\
\begin{aligned}
&\left(H_{\mathrm{I}}\left(H_{0}^{\prime}-E_{0}\right)^{-1} Q_{0} H_{\mathrm{I}} \Psi_{0}\right)^{(2)}\left(k_{1}, k_{2}\right)= \frac{1}{2 \sqrt{2}} \sum_{\ell, j=1}^{J}\left\{g_{\ell}\left(k_{2}\right) g_{j}\left(k_{1}\right) B_{\ell}\left(\widetilde{A}+\omega\left(k_{1}\right)\right)^{-1} B_{j} \psi_{0}\right. \\
&\left.+g_{\ell}\left(k_{1}\right) g_{j}\left(k_{2}\right) B_{\ell}\left(\widetilde{A}+\omega\left(k_{2}\right)\right)^{-1} B_{j} \psi_{0}\right\} \\
& k_{1}, k_{2} \in \mathbb{R}^{\nu}, \omega\left(k_{1}\right)>0, \omega\left(k_{2}\right)>0,
\end{aligned} \\
\left(H_{\mathrm{I}}\left(H_{0}^{\prime}-E_{0}\right)^{-1} Q_{0} H_{\mathrm{I}} \Psi_{0}\right)^{(n)}=0, \quad n \neq 0,2 .
\end{gathered}
$$

Similarly we see that $\left(H_{0}^{\prime}-E_{0}\right)^{-1} Q_{0} H_{\mathrm{I}} \Psi_{0}$ is in $D\left(H_{0}\right)$ with

$$
\begin{aligned}
& \left(H_{0}\left(H_{0}^{\prime}-E_{0}\right)^{-1} Q_{0} H_{\mathrm{I}} \Psi_{0}\right)^{(1)}(k)=\frac{1}{\sqrt{2}} \sum_{j=1}^{J}\left(g_{j}(k)+E_{0} g_{j}(k)(\widetilde{A}+\omega(k))^{-1}\right) B_{j} \psi_{0}, \\
& \left(H_{0}\left(H_{0}^{\prime}-E_{0}\right)^{-1} Q_{0} H_{\mathrm{I}} \Psi_{0}\right)^{(n)}=0, \quad n \neq 1 .
\end{aligned}
$$

Thus the desired result follows.

Lemma 5.10 Assume (A.1)-(A.6). Let

$$
\begin{aligned}
& N_{\mathrm{GSB}}:=\frac{1}{2} \sum_{j, \ell=1}^{J} \int_{\omega(k)>0}\left\langle(\widetilde{A}+\omega(k))^{-1} B_{j} \psi_{0},(\widetilde{A}+\omega(k))^{-1} B_{\ell} \psi_{0}\right\rangle g_{j}(k)^{*} g_{\ell}(k) d k, \\
& a_{\mathrm{GSB}}:=\frac{1}{2} \sum_{j, \ell=1}^{J} \int_{\omega(k)>0}\left\langle B_{j} \psi_{0},(\widetilde{A}+\omega(k))^{-1} B_{\ell} \psi_{0}\right\rangle g_{j}(k)^{*} g_{\ell}(k) d k .
\end{aligned}
$$

Then $a_{\mathrm{GSB}}>0$ and

$$
E_{0}(\lambda) \leq E_{0}-\frac{a_{\mathrm{GSB}} \lambda^{2}}{1+N_{\mathrm{GSB}} \lambda^{2}}
$$

In particular, for all $\lambda \in \mathbb{I}_{r}^{\times}, E_{0}(\lambda)<E_{0}$ and $E_{0}(\lambda) \in \rho\left(H_{0}\right)$.

Proof. As already seen, in the GSB model, (2.19) holds. Hence, by Lemmas 5.6 and 5.9 , we can apply Lemma2.7. In the present case, we have $N_{0}=N_{\mathrm{GSB}}, a=a_{\mathrm{GSB}}$ and $b=0$. Thus the desired result follows.

Lemma 5.11 Assume (A.1)-(A.5) and let $\Psi_{0}(\lambda)$ be as in Theorem 5.5. Then, for all $\lambda \in \mathbb{I}_{r}^{\times}, \Psi_{0}(\lambda)$ overlaps with $\Psi_{0}$ and

$$
\lim _{\lambda \rightarrow 0}\left|\left\langle\Psi_{0}, \Psi_{0}(\lambda)\right\rangle\right|=1
$$


Proof. By (A.5), we have $p_{0} \otimes P_{\Omega_{0}} \Psi_{0}(\lambda)=\left\langle\Psi_{0}, \Psi_{0}(\lambda)\right\rangle \Psi_{0}$. Hence, by Theorem 5.5,

$$
1 \geq\left|\left\langle\Psi_{0}, \Psi_{0}(\lambda)\right\rangle\right|^{2} \geq 1-\lambda^{2} M^{2}>0
$$

Hence the desired results follow.

Let (A.1)-(A.6) be satisfied. Then, by Lemma 5.11, we can define for all $\lambda \in \mathbb{I}_{r}^{\times}$

$$
\Psi_{\mathrm{GSB}}(\lambda):=\frac{1}{\left\langle\Psi_{0}, \Psi_{0}(\lambda)\right\rangle} \Psi_{0}(\lambda) .
$$

Then, as in (3.1) and (3.2), we have for all $\lambda \in \mathbb{I}_{r}^{\times}$

$$
\begin{aligned}
& E_{0}(\lambda)=E_{0}+\lambda\left\langle\Psi_{0}, H_{\mathrm{I}} \Psi_{\mathrm{GSB}}(\lambda)\right\rangle, \\
& \Psi_{\mathrm{GSB}}(\lambda)=\Psi_{0}+\lambda\left(E_{0}(\lambda)-H_{0}\right)^{-1} Q_{0} H_{\mathrm{I}} \Psi_{\mathrm{GSB}}(\lambda) .
\end{aligned}
$$

Lemma 5.12 Condition (H.4) with $\Psi(\lambda)=\Psi_{\mathrm{GSB}}(\lambda)$ holds:

$$
\lim _{\lambda \rightarrow 0}\left\|\Psi_{\mathrm{GSB}}(\lambda)\right\|=1 .
$$

Proof. By Theorem 5.5-(ii) and $\left\|p_{0} \otimes P_{0}\right\|=1$, we have

$$
1 \geq\left\|\Psi_{0}(\lambda)\right\| \geq \sqrt{1-\lambda^{2} M^{2}} .
$$

Hence $\lim _{\lambda \rightarrow 0}\left\|\Psi_{0}(\lambda)\right\|=1$. By this fact and (5.32), we obtain (5.36).

Thus we have shown that, under assumptions (A.1)-(A.6), conditions (H.1)-(H.5) in Section 3 hold. Therefore, to establish asymptotic expansions of $E_{0}(\lambda)$, we need only to check assumptions in Theorem 4.1 other than (H.1)-(H.5).

For an independent interest, we first derive the asymptotic expansion of $E_{0}(\lambda)$ up to the second order in $\lambda$.

Theorem 5.13 Assume (A.1)-(A.6). Then

$$
E_{0}(\lambda)=E_{0}-a_{\mathrm{GSB}} \lambda^{2}+o\left(\lambda^{2}\right) \quad(\lambda \rightarrow 0),
$$

where $a_{\mathrm{GSB}}$ is given by (5.30).

Proof. We need only to check (3.14).

It follows from (5.20) that $H_{\mathrm{I}} \Psi_{0} \in D(G(\lambda))$ and

$$
\begin{aligned}
& \left(G(\lambda) H_{\mathrm{I}} \Psi_{0}\right)^{(0)}=\frac{1}{2} \sum_{\ell=1}^{J} \sum_{j=1}^{J} \int_{\mathbb{R}^{\nu}} d k g_{\ell}(k)^{*} g_{j}(k) B_{\ell}\left(E(\lambda)-E_{0}-\widetilde{A}-\omega(k)\right)^{-1} B_{j} \psi_{0}, \\
& \begin{array}{r}
\left(G(\lambda) H_{\mathrm{I}} \Psi_{0}\right)^{(2)}\left(k_{1}, k_{2}\right)=\frac{1}{2 \sqrt{2}} \sum_{\ell=1}^{J} \sum_{j=1}^{J}\left\{g_{\ell}\left(k_{2}\right) g_{j}\left(k_{1}\right) B_{\ell}\left(E(\lambda)-E_{0}-\widetilde{A}-\omega\left(k_{1}\right)\right)^{-1} B_{j} \psi_{0}\right. \\
\left.\quad+g_{\ell}\left(k_{1}\right) g_{j}\left(k_{2}\right) B_{\ell}\left(E(\lambda)-E_{0}-\widetilde{A}-\omega\left(k_{2}\right)\right)^{-1} B_{j} \psi_{0}\right\},
\end{array} \\
& \left(G(\lambda) H_{\mathrm{I}} \Psi_{0}\right)^{(n)}=0, \quad n \neq 0,2 .
\end{aligned}
$$


By (5.26) and $E(\lambda)<E_{0}$, we have

$$
\begin{aligned}
&\left\|\left(G(\lambda) H_{\mathrm{I}} \Psi_{0}\right)^{(0)}\right\| \leq \frac{1}{2} \sum_{\ell=1}^{J} \sum_{j=1}^{J} \int_{\mathbb{R}^{\nu}} d k\left|g_{\ell}(k)\right|\left|g_{j}(k)\right|\left(a \varepsilon+b_{\varepsilon} \omega(k)^{-1}\right)\left\|B_{j} \psi_{0}\right\|, \\
&\left\|\left(G(\lambda) H_{\mathrm{I}} \Psi_{0}\right)^{(2)}\left(k_{1}, k_{2}\right)\right\| \leq \frac{1}{2 \sqrt{2}} \sum_{\ell=1}^{J} \sum_{j=1}^{J}\left\{\left|g_{\ell}\left(k_{2}\right)\right|\left|g_{j}\left(k_{1}\right)\right|\left(a \varepsilon+b_{\varepsilon} \omega\left(k_{1}\right)^{-1}\right)\left\|B_{j} \psi_{0}\right\|\right. \\
&+\left|g_{\ell}\left(k_{1}\right)\left\|g_{j}\left(k_{2}\right) \mid\left(a \varepsilon+b_{\varepsilon} \omega\left(k_{2}\right)^{-1}\right)\right\| B_{j} \psi_{0} \|\right\} .
\end{aligned}
$$

These estimates imply (3.14).

We next consider the asymptotic expansion of $E_{0}(\lambda)$ in $\lambda$ up to the $N$-th order with $N \geq 3$.

Lemma 5.14 Let $N \geq 2$ be a natural number and suppose that

$$
g_{j}, \frac{g_{j}}{\omega^{N-1}} \in L^{2}\left(\mathbb{R}^{\nu}\right), \quad j=1, \ldots, J
$$

Then (4.5) holds in the present model.

Remark 5.15 If (5.38) holds, then $g_{j} / \omega^{s} \in L^{2}\left(\mathbb{R}^{\nu}\right)$ for all $s \in[0, N-1]$.

Proof. Let $q=1, \ldots, N-1$ and $r_{\alpha}=0,1, \ldots, N-2(\alpha=1, \ldots, q)$. By assumption (5.38) and Remark 5.15, one sees that $\Psi_{0}$ is in $D\left(\left(H_{0}^{\prime}-E_{0}\right)^{-\left(r_{q-1}+1\right)} Q_{0} H_{\mathrm{I}}\left(H_{0}^{\prime}-\right.\right.$ $\left.\left.E_{0}\right)^{-\left(r_{q}+1\right)} Q_{0} H_{\mathrm{I}}\right)$ and the vector

$$
\Psi_{2}:=\left(H_{0}^{\prime}-E_{0}\right)^{-\left(r_{q-1}+1\right)} Q_{0} H_{\mathrm{I}}\left(H_{0}^{\prime}-E_{0}\right)^{-\left(r_{q}+1\right)} Q_{0} H_{\mathrm{I}} \Psi_{0}
$$

takes the following form:

$$
\Psi_{2}=\frac{1}{2} \sum_{j, \ell=1}^{J}\left(H_{0}^{\prime}-E_{0}\right)^{-\left(r_{q-1}+1\right)} B_{\ell} \otimes a\left(g_{\ell}\right)^{*}\left(H_{0}^{\prime}-E_{0}\right)^{-\left(r_{q}+1\right)} B_{j} \otimes a\left(g_{j}\right)^{*} \Psi_{0}
$$

Hence, under the natural identification $(5.3), \Psi_{2}$ is in the 2-boson space $\mathcal{H}^{(2)}$ with

$$
\begin{aligned}
& \Psi_{2}\left(k_{1}, k_{2}\right) \\
& =\frac{1}{2} \sum_{j, \ell=1}^{J}\left\{\left(\widetilde{A}+\omega\left(k_{1}\right)+\omega\left(k_{2}\right)\right)^{-\left(r_{q-1}+1\right)} g_{j}\left(k_{1}\right) g_{\ell}\left(k_{2}\right) B_{\ell}\left(\widetilde{A}+\omega\left(k_{1}\right)\right)^{-\left(r_{q}+1\right)}\right. \\
& \left.\times B_{j} \psi_{0}+\left(\widetilde{A}+\omega\left(k_{1}\right)+\omega\left(k_{2}\right)\right)^{-\left(r_{q-1}+1\right)} g_{j}\left(k_{2}\right) g_{\ell}\left(k_{1}\right) B_{\ell}\left(\widetilde{A}+\omega\left(k_{2}\right)\right)^{-\left(r_{q}+1\right)} B_{j} \psi_{0}\right\}
\end{aligned}
$$


for a.e. $\left(k_{1}, k_{2}\right) \in\left(\mathbb{R}^{\nu}\right)^{2}$. For $q \geq 3$ and $\alpha=1, \ldots, q$, we have

$$
\begin{aligned}
& \left(\prod_{\alpha=1}^{q}\left(H_{0}^{\prime}-E_{0}\right)^{-\left(r_{\alpha}+1\right)} Q_{0} H_{\mathrm{I}}\right) P_{0} \\
& =\frac{1}{\sqrt{2}} \sum_{j_{1}, \ldots, j_{q}=1}^{J}\left(\widetilde{A}+H_{\mathrm{b}}^{\prime}\right)^{-\left(r_{1}+1\right)} Q_{0} B_{j_{1}} \otimes a\left(g_{j_{1}}\right)^{\#}\left(\widetilde{A}+H_{\mathrm{b}}^{\prime}\right)^{-\left(r_{2}+1\right)} Q_{0} B_{j_{2}} \otimes a\left(g_{j_{2}}\right)^{\#} \\
& \cdots Q_{0} B_{j_{q-2}} \otimes a\left(g_{j_{q-2}}\right)^{\#}\left(H_{0}^{\prime}-E_{0}\right)^{-\left(r_{q-1}+1\right)} B_{j_{q-1}} \otimes a\left(g_{j_{q-1}}\right)^{*} \\
& \times\left(H_{0}^{\prime}-E_{0}\right)^{-\left(r_{q}+1\right)} B_{j_{q}} \otimes a\left(g_{j_{q}}\right)^{*} P_{0} .
\end{aligned}
$$

Note that, because of the action of $Q_{0}$, the last two $a(\cdot)^{\# \text { 's }}$ on the right hand side are $a(\cdot)^{*}$.

As in Lemma 5.8, we can show that, for all $\Phi \in D\left(H_{\mathrm{b}}^{\prime-1}\right)$ and $n \geq 1$

$$
\begin{aligned}
& \left\|\left(B_{j}\left(\widetilde{A}+H_{\mathrm{b}}^{\prime}\right)^{-1} \Phi\right)^{(n)}\left(k_{1}, \ldots, k_{n}\right)\right\| \\
& \leq a \varepsilon\left\|\Phi^{(n)}\left(k_{1}, \ldots, k_{n}\right)\right\|+b_{\varepsilon} \frac{1}{\sum_{i=1}^{n} \omega\left(k_{i}\right)}\left\|\Phi^{(n)}\left(k_{1}, \ldots, k_{n}\right)\right\|
\end{aligned}
$$

for a.e. $\left(k_{1}, \ldots, k_{n}\right) \in\left(\mathbb{R}^{\nu}\right)^{n}$, where $\|\cdot\|$ denotes the norm of $\mathcal{K}$ and $\varepsilon>0$ is arbitrary.

For all $f \in L^{2}\left(\mathbb{R}^{\nu}\right)$, the operator $a(f)$ (resp. $\left.a(f)^{*}\right)$ maps the $n$-boson space $\mathcal{H}^{(n)}$ to $\mathcal{H}^{(n-1)}\left(\operatorname{resp} . \mathcal{H}^{(n+1)}\right)$ and, for all $p \geq 1,\left(\widetilde{A}+H_{\mathrm{b}}^{\prime}\right)^{-p}$ maps $D\left(\left(\widetilde{A}+H_{\mathrm{b}}^{\prime}\right)^{-p}\right) \cap \mathcal{H}^{(n)}$ into $\mathcal{H}^{(n)}$ with

$$
\begin{array}{r}
\left(\left(\widetilde{A}+H_{\mathrm{b}}^{\prime}\right)^{-p} \Psi\right)^{(n)}\left(k_{1}, \ldots, k_{n}\right)=\left(\widetilde{A}+\sum_{j=1}^{n} \omega\left(k_{j}\right)\right)^{-p} \Psi^{(n)}\left(k_{1}, \ldots, k_{n}\right) \in \mathcal{K} \\
\text { a.e. }\left(k_{1}, \ldots, k_{n}\right) \in\left(\mathbb{R}^{\nu}\right)^{n}
\end{array}
$$

for all $\Psi \in D\left(\left(\widetilde{A}+H_{\mathrm{b}}^{\prime}\right)^{-p}\right) \cap \mathcal{H}^{(n)}$. Moreover, for all $\Psi \in D\left(\left(\widetilde{A}+H_{\mathrm{b}}^{\prime}\right)^{-p}\right) a(f) B_{j}(\widetilde{A}+$ $\left.\left.\left.H_{\mathrm{b}}^{\prime}\right)^{-1}\right) \cap D\left(\left(\widetilde{A}+H_{\mathrm{b}}^{\prime}\right)^{-p}\right) a(f)^{*} B_{j}\left(\widetilde{A}+H_{\mathrm{b}}^{\prime}\right)^{-1}\right) \cap \mathcal{H}^{(n)}$, the following hold:

$$
\begin{aligned}
& \left(\left(\widetilde{A}+H_{\mathrm{b}}^{\prime}\right)^{-p} a(f)^{*} B_{j}\left(\widetilde{A}+H_{\mathrm{b}}^{\prime}\right)^{-1} \Psi\right)^{(n+1)}\left(k_{1}, \ldots, k_{n+1}\right) \\
& =\frac{1}{\sqrt{n+1}}\left(\widetilde{A}+\sum_{j=1}^{n+1} \omega\left(k_{j}\right)\right)^{-p} \sum_{i=1}^{n} f\left(k_{i}\right)\left(B_{j}\left(\widetilde{A}+H_{\mathrm{b}}^{\prime}\right)^{-1} \Psi\right)^{(n)}\left(k_{1}, \ldots, \hat{k}_{i}, \ldots, k_{n+1}\right), \\
& \left(\left(\widetilde{A}+H_{\mathrm{b}}^{\prime}\right)^{-p} a(f) B_{j}\left(\widetilde{A}+H_{\mathrm{b}}^{\prime}\right)^{-1} \Psi\right)^{(n-1)}\left(k_{1}, \ldots, k_{n-1}\right) \\
& =\sqrt{n}\left(\widetilde{A}+\sum_{i=1}^{n-1} \omega\left(k_{i}\right)\right)^{-p} \int_{\mathbb{R}^{\nu}} d k_{n} f\left(k_{n}\right)^{*}\left(B_{j}\left(\widetilde{A}+H_{\mathrm{b}}^{\prime}\right)^{-1} \Psi\right)^{(n)}\left(k_{1}, \ldots, k_{n-1}, k_{n}\right),
\end{aligned}
$$


where $\hat{k}_{i}$ indicates the omission of $\hat{k}_{i}$. Hence, by (5.40), we have

$$
\begin{aligned}
& \left\|\left(\left(\widetilde{A}+H_{\mathrm{b}}^{\prime}\right)^{-p} a(f)^{*} B_{j}\left(\widetilde{A}+H_{\mathrm{b}}^{\prime}\right)^{-1} \Psi\right)^{(n+1)}\left(k_{1}, \ldots, k_{n+1}\right)\right\| \\
& \leq \frac{1}{\sqrt{n+1}}\left(\sum_{j=1}^{n+1} \omega\left(k_{j}\right)\right)^{-p} \sum_{i=1}^{n+1}\left|f\left(k_{i}\right)\right|\left\{a \varepsilon+b_{\varepsilon} \frac{1}{\sum_{h=1, h \neq i}^{n+1} \omega\left(k_{h}\right)}\right\} \\
& \quad \times\left\|\Psi^{(n)}\left(k_{1}, \ldots, \hat{k}_{i}, \ldots, k_{n+1}\right)\right\|,
\end{aligned}
$$

By these estimates and (5.39), one can see that $\Psi_{0}$ is in $D\left(\prod_{\alpha=1}^{q}\left(H_{0}^{\prime}-E_{0}\right)^{-\left(r_{\alpha}+1\right)} Q_{0} H_{\mathrm{I}}\right)$. Thus the desired result follows.

Remark 5.16 The condition $g_{j} / \omega \in L^{2}\left(\mathbb{R}^{\nu}\right)(j=1, \ldots, J)$ is called the infrared regular condition in the GSB model (if this condition is not satisfied for some $j$, then $H_{\mathrm{GSB}}$ may have no ground states [7]). Condition (5.38) with $N \geq 3$ means that $g_{j}$ is more infrared regular than the case $N=2$. On the other hand, (4.5) expresses a regularity of $\Psi_{0}$ in terms of $H_{0}$ and $H_{\mathrm{I}}$ in the sense to which operator domains it belongs. Hence Lemma 5.14 shows that the regularity of $\Psi_{0}$ in the sense just mentioned is closely related to the infrared behavior of cutoff functions $g_{j}(j=1, \ldots, J)$.

Theorem 5.17 Assume (A.1)-(A.6) and (5.38) with $N \geq 4$ even. Let $b_{n}(n \geq 1)$ be the number $a_{n}$ defined by (4.4) with $Q_{0}, H_{0}, H_{\mathrm{I}}$ and $\Psi_{0}$ replaced by those in the GSB model. Then

$$
b_{2 n-1}=0, \quad n=1, \ldots, \frac{N}{2}
$$

and

$$
E_{0}(\lambda)=E_{0}+\sum_{n=1}^{N / 2} b_{2 n} \lambda^{2 n}+o\left(\lambda^{N}\right) \quad(\lambda \rightarrow 0) .
$$

Proof. By the discussions made so far and Lemma 5.14, to prove that $E_{0}(\lambda)$ has an asymptotic expansion up to $N$-th order in $\lambda$, we need only to show that, in the present case, (4.8) holds. We first do it. We have

$$
H_{0}^{\prime}-E(\lambda)=\widetilde{A}+H_{\mathrm{b}}^{\prime}+E_{0}-E(\lambda)
$$

and $E_{0}-E(\lambda)>0$. Hence, in the same way as in the proof of Lemma 5.14, we can show 
that, for all $\Psi \in D(G(\lambda)) \cap \mathcal{H}^{(n)}$,

$$
\begin{aligned}
& \left\|\left(a(f)^{*} B_{j}\left(E(\lambda)-H_{0}^{\prime}\right)^{-1} \Psi\right)^{(n+1)}\left(k_{1}, \ldots, k_{n+1}\right)\right\| \\
& \leq \frac{1}{\sqrt{n+1}} \sum_{i=1}^{n+1}\left|f\left(k_{i}\right)\right|\left\{a \varepsilon+b_{\varepsilon} \frac{1}{\sum_{h=1, h \neq i}^{n+1} \omega\left(k_{h}\right)}\right\} \\
& \quad \times\left\|\Psi^{(n)}\left(k_{1}, \ldots, \hat{k}_{i}, \ldots, k_{n+1}\right)\right\|, \\
& \left\|\left(a(f) B_{j}\left(E(\lambda)-H_{0}^{\prime}\right)^{-1} \Psi\right)^{(n-1)}\left(k_{1}, \ldots, k_{n-1}\right)\right\| \\
& \leq \sqrt{n} \int_{\mathbb{R}^{\nu}} d k_{n}\left|f\left(k_{n}\right)\right|\left\{a \varepsilon+b_{\varepsilon} \frac{1}{\sum_{h=1}^{n} \omega\left(k_{h}\right)}\right\}\left\|\Psi^{(n)}\left(k_{1}, \ldots, \ldots, k_{n}\right)\right\| .
\end{aligned}
$$

By repeating this type of estimates, we see that (4.8) holds. Therefore, by Theorem 4.1, we obtain (4.9) with $a_{n}$ replaced by $b_{n}$. By Theorem $5.1, E_{0}(\lambda)$ is even in $\lambda$. This implies (5.41).

Remark 5.18 We can prove (5.41) by induction in $n$, too.

Remark 5.19 The methods described above would be applied also to other models [4] of a quantum system interacting with a massless quantum field .

\section{Acknowledgement}

This work is supported by Grant-in-Aid 24540154 for Scientific Research from JSPS.

\section{References}

[1] A. Abdesselam, The ground state energy of the massless spin-boson model, Ann. Henri Poincaré 12 (2011), 1321-1347.

[2] A. Abdesselam and D. Hasler, Analyticity of the ground state energy for massless Nelson models, Commu. Math. Phys. 310 (2012), 511-536.

[3] A. Arai, Essential spectrum of a self-adjoint operator on an abstract Hilbert space of Fock type and applications to quantum field Hamiltonians, J. Math. Anal. Appl. 246 (2000), 189-216.

[4] A. Arai, Mathematical theory of quantum particles interacting with a quantum field, in Non-Commutativity, Infinite-Dimensionality and Probability at the Crossroads (Editors: N. Obata, T. Matsui and A. Hora), World Scientific, 2002, Singapore, $1-50$. 
[5] A. Arai and M. Hirokawa, On the existence and uniqueness of ground states of a generalized spin-boson model, J. Funct. Anal. 151 (1997), 455-503.

[6] A. Arai and M. Hirokawa, Stability of ground states in sectors and its application to the Wigner-Weisskopf model, Rev. Math. Phys. 13 (2001), 513-527.

[7] A. Arai, M. Hirokawa and F. Hiroshima, On the absence of eigenvalues of Hamiltonians in a class of massless quantum field models without infrared cutoff, J. Funct. Anal. 168 (1999), 470-497.

[8] V. Bach, J. Fröhlich and I. M. Sigal, Renormalization group analysis of spectral problems in quantum field theory, Adv. in Math. 137 (1998), 205-298.

[9] V. Bach, J. Fröhlich and I. M. Sigal, Quantum electrodynamics of confined nonrelativistic particles, Adv. in Math. 137 (1998), 299-395.

[10] L. Brillouin, Champs self-consistents et electrons metalliques - III, J. de Phys. Radium 4 (1933), 1-9.

[11] J. Dereziński and C. Gérard, Asymptotic completeness in quantum field theory. Massive Pauli-Fierz Hamiltonians, Rev. Math. Phys. 11 (1999), 383-450.

[12] M. Griesemer and D. Hasler, Analytic perturbation theory and renormalization analysis of matter coupled to quantized radiation, Ann. Henri Poincaré 10 (2009), 577621.

[13] D. Hasler and I. Herbst, Ground states in the spin boson model, Ann. Henri Poincaré 12 (2011), 621-677.

[14] F. Hiroshima, Analysis of ground states of atoms interacting with a quantized radiation field, in Topics in the Theory of Schrödinger Operators (Editors: H. Araki and H. Ezawa), World Scientific, 2004, Singapore, Chapter 5.

[15] T. Kato, Perturbation Theory for Linear Operators, Second Edition, Springer, Berlin, Heidelberg, 1976.

[16] E. Nelson, Interaction of nonrelativistic particles with a quantized scalar field, $J$. Mah. Phys. 5 (1964), 1190-1197.

[17] M. Reed and B. Simon, Methods of Modern Mathematical Physics II: Fourier Analysis, Self-adjointness, Academic Press, New York, 1975.

[18] M. Reed and B. Simon, Methods of Modern Mathematical Physics IV: Analysis of Operators, Academic Press, New York, 1978. 
[19] E. P. Wigner, On a modification of the Rayleigh-Schrödinger perturbation theory, Magyar Tudományos Akadémia Matematikai és Természettudományi Értesitöje $\mathbf{5 3}$ (1935), 477-482. A. S. Wightman (Ed.), Collected Works of Eugene Paul Wigner Part A Volume IV, pp. 131-136, Springer, Berlin, Heidelberg, 1997.

[20] J. M. Ziman, Elements of Advanced Quantum Theory, Cambridge University Press, 1969. 